\title{
Modelling of Bingham and Herschel-Bulkley flows with mixed P1/P1 finite elements stabilized with orthogonal subgrid scale
}

\author{
Elvira Moreno ${ }^{\mathrm{a}}$, Antonia Larese ${ }^{\mathrm{b}, *}$, Miguel Cervera ${ }^{\mathrm{b}}$ \\ a Departamento de Ordenación de Cuencas, Ingeniería Forestal, Universidad de los Andes, ULA, Vía Chorros de Milla, 5101, Mérida Venezuela \\ ${ }^{\mathrm{b}}$ International Center for Numerical Methods in Engineering (CIMNE), Technical University of Catalonia (UPC), Edificio C1, Campus Norte, Jordi Girona 1-3, \\ 08034, Barcelona Spain
}

\section{A R T I C L E I N F O}

\section{Article history:}

Received 3 August 2015

Revised 27 November 2015

Accepted 12 December 2015

Available online 19 December 2015

Keywords:

Bingham flows

Herschel-Bulkley flows

Variational multiscale stabilization

Orthogonal subscale stabilization

Moving cylinder

Extrusion

\begin{abstract}
A B S T R A C T
This paper presents the application of a stabilized mixed pressure/velocity finite element formulation to the solution of viscoplastic non-Newtonian flows. Both Bingham and Herschel-Bulkley models are considered.

The detail of the discretization procedure is presented and the Orthogonal Subgrid Scale (OSS) stabilization technique is introduced to allow for the use of equal order interpolations in a consistent way. The matrix form of the problem is given.

A series of examples is presented to assess the accuracy of the method by comparison with the results obtained by other authors. The extrusion in a Bingham fluid and the movement of a moving and rotating cylinder are analyzed in detail. The evolution of the streamlines, the yielded and unyielded regions, the drag and lift forces are presented.
\end{abstract}

These benchmark examples show the capacity of the mixed OSS formulation to reproduce the behavior of a Bingham and Herschel-Bulkley flows with the required accuracy.

(c) 2015 Elsevier B.V. All rights reserved.

\section{Introduction}

The aim of this paper is to present a continuum formulation and its correspondent discrete version for Bingham and HerschelBulkley confined flows, using mixed velocity/pressure linear finite elements.

Bingham and Herschel-Bulkley are viscoplastic non-Newtonian fluids characterized by the presence of a threshold stress, the yield stress. When the yield stress is exceeded the fluid flows; contrariwise, if this limit is not achieved, the fluid acts as a rigid material [35].

Bingham plastics are very common in industry. They can model the behavior of a large number of materials, such as paints, and many products in food industry (ketchup, mayonnaise, etc). Bingham conceived this rheological law while studying the behavior of paints at the beginning of XX century [10]. The Herschel-Bulkley model is a generalization of the Bingham one, and it is less known. It describes the behavior of pastes, gels, or drilling fluids. It can be also used for simulating debris flow ([64,79]). Both models have a severe discontinuity in their rheological behavior due to the ex-

\footnotetext{
* Corresponding author. Tel.: +34 9340107 94; fax +34 934016517

E-mail address: antoldt@cimne.upc.edu (A. Larese).
}

istence of the threshold yield stress, which poses some numerical difficulties.

The different procedures proposed in the literature to deal with the discontinuity problem can be classified in two main categories: a variational reformulation using multipliers, or a regularization of the constitutive law. In the first case, the nonlinear problem can be rewritten in the form of a variational inequality model, following the original work by Duvaut and Lions [40]. This is equivalent to the existence of a symmetric second order tensor, called multiplier, whose value defines the rigid (if smaller than one) or yielded zone (if equal to one). This approach was recently used together with operator-splitting methods to numerically solve the problem $([37,52,53,83])$. It can be demonstrated [42] that the solution of the constrained variational inequality is equivalent to a minimization problem of an equivalent variational equality form. Many authors solve this problem using augmented Lagrangian approaches $([43,92])$. Usually these formulations are designed for finite elements, but a finite difference model can be found in [71]. For a comprehensive review on both operator-splitting methods and augmented Lagrangian approaches, the consultation of $[42,51]$ is recommended.

In the second case, a regularized constitutive model is used. Different regularized formulations have been proposed: Bercovier 
and Engelman [7], Tanner and Milthorpe [88], and Beris [8], among others. Tanner and Milthorpe were the first to propose a double viscosity model, while Beris used a Von Mises yield criterion in the unyielded zone and an ideal Bingham model in the yielded region. In 1987, Papanastasiou [73] proposed a regularization valid both for the unyielded and the yielded regions. Recently, Souza Mendes and Dutra (SMD) [39] presented a modification to the model by Papanastasiou. Among the most commonly used models, Figaard and Nouar [41] prove that Papanastasiou's model provides a better convergence to the exact solution.

The main reason for regularizing the discontinuity of the exact viscoplastic behavior is to allow its direct implementation in standard numerical solvers.

The movement of isothermal flows is governed by conservation of linear momentum and mass, represented by the Navier-Stokes equations. In the case of non-Newtonian fluids, the constitutive law has a variable viscosity whose behavior is given by the rheological models.

Traditionally viscoplastic flows are calculated using finite elements $([1,70,73,93])$ but an attempt to use finite volumes was proposed by Bharti et al. [9], and Tanner and Milthorpe [88] used boundary elements.

In this work, a mixed velocity/pressure finite element formulation for simplicial elements is developed. This means that both velocity and pressure are interpolated piecewise linearly within the finite element mesh. This is a frequent choice in fluid dynamics because of their simplicity. On the one hand, this kind of linear elements, called $P 1 / P 1$, present a source of instability due to the combination of the interpolation spaces of pressure and velocity [29]. The Ladyzenskaja-Babuška-Brezzi condition is not satisfied in such incompressible problem and spurious oscillations of the pressure can compromise the solution [13]. On the other hand, the convective term presents another source of instability for convection-dominated problems. The use of a proper stabilization technique is therefore needed to ensure stability and convergence of the solution.

Nowadays the most effective stabilization techniques are based on the concept of sub-scales. These were first introduced by Hughes [49], who proposed an Algebraic Sub-Grid Scale (ASGS) technique for the stabilization of a scalar diffusion-reaction equation. Codina generalized the approach for multi-dimensional systems [30]. The idea is to split the unknown in a part that can be solved by the finite element approximation plus an unresolvable scale (i.e. the sub-scale) that cannot be captured by the finite element discretization. The sub-scale is approximated in a consistent residual fashion so that its variational stabilizing effect is captured. More recently, Codina proposed to use a space orthogonal to the finite element space for the subscale, introducing the Orthogonal Subgrid Scale (OSS) stabilization technique ([31,32]). The main advantage of OSS is that it guarantees minimal numerical dissipation on the discrete solution, because it adds nothing to those components of the residual already belonging to the FE subspace. This maximizes accuracy for a given mesh, an issue always important and no less in nonlinear problems.

OSS has been successfully applied to the Stokes problem, to the convection-diffusion-reaction equations and to the Navier-Stokes equations. Nowadays it is used in a wide range of different problems in fluid dynamics $([30,31,34,56-58,76,81])$ and solid mechanics ([17-22,27,28]). Castillo and Codina presented a three fields formulation for visco-elastic [16], power law and Carreau-Yasuda [15] fluids comparing ASGS and OSS. In the present work, the OSS stabilization technique is applied to the Navier-Stokes equations to model regularized Bingham and Herschel-Bulkley flows.

The structure of the paper is as follows. First, both the Bingham and the Herschel-Bulkley models are presented. An overview of the regularizations proposed in the literature is given. The governing equations for a non-Newtonian fluid are presented in their strong form. The corresponding discrete model is presented and the stabilization using Orthogonal Subgrid Scales (OSS) is explained in detail. The matrix form of the problem is given. Secondly, the Bingham model is applied to two well known problems: an extrusion process and a cylinder moving in a Bingham fluid confined between two parallel planes. Then, a cylinder moving in an Herschel-Bulkley fluid is modeled in two different scenarios: a cylinder moving with constant velocity and a cylinder moving and rotating around its axis. In all the cases the solution is compared with available results from other authors. Finally, some conclusions on the performance of the proposed formulation are given.

\section{Viscoplastic fluids}

In the present work, viscoplastic fluids are considered. These are characterized by the existence of a threshold stress, the yield stress $\left(\tau_{y}\right)$, which must be exceeded for the fluid to deform. For lower values of stress the viscoplastic fluids are completely rigid or can show some sort of elasticity. Once the yield stress is reached and exceeded, viscoplastic fluids may exhibit a Newtonian-like behavior with constant viscosity (Bingham plastics fluids) or with rate dependent viscosity (Herschel-Bulkley fluids among others).

Let us introduce, for later use, the equivalent strain rate $\dot{\gamma}$ and the equivalent deviatoric stress $\tau$ in terms of the second invariants of the rate of strain tensor ( $\boldsymbol{\varepsilon}=\nabla^{s} \mathbf{u}$, being $\nabla^{s} \mathbf{u}$ the symmetric part of the velocity gradient) and of the deviatoric part of the stress tensor ( $\boldsymbol{\tau}=2 \mu \boldsymbol{\varepsilon}(\mathbf{u})$, being $\mu$ the viscosity), respectively:

$\dot{\gamma}=(2 \varepsilon: \varepsilon)^{\frac{1}{2}} \quad \tau=\left(\frac{1}{2} \boldsymbol{\tau}: \boldsymbol{\tau}\right)^{\frac{1}{2}}$

\subsection{Herschel-Bulckley and Bingham fluids}

The Herschel-Bulkley model [46] combines the existence of a yield stress with a power law model for the viscosity

$$
\begin{aligned}
& \mu(\dot{\gamma})=k \dot{\gamma}^{n-1}+\frac{\tau_{y}}{\dot{\gamma}} \text { if } \tau \geq \tau_{y} \\
& \dot{\gamma}=0 \text { if } \tau<\tau_{y}
\end{aligned}
$$

where $k$ is the consistency parameter and $n$ is the flow index. The yield stress needs to be overcome for the material to flow. When the yield stress is exceeded, the material flows with a nonlinear relation between stress and rate of strain as in a pseudoplastic fluid, if $n>1$, or a dilatant one, if $n<1$.

The deviatoric stress tensor is therefore

$$
\begin{aligned}
& \boldsymbol{\tau}=2\left(k \dot{\gamma}^{n-1}+\frac{\tau_{y}}{\dot{\gamma}}\right) \boldsymbol{\varepsilon}(\boldsymbol{u}) \text { if } \tau \geq \tau_{y} \\
& \dot{\gamma}=0 \text { if } \tau<\tau_{y}
\end{aligned}
$$

When the rate of deformation tends to zero this ideal rheological model presents a singularity and the viscosity tends to infinity $\left(\lim _{\dot{\gamma} \rightarrow 0} \mu(\dot{\gamma})=\infty\right)$. This aspect is a serious inconvenient when treating the model numerically ([11]). For this reason, many authors have proposed regularized versions of the Herschel-Bulkley model, such as the double viscosity Tanner and Milthrope model [88], the widely used Papanastasiou regularized model [73], or the Souza Mendes and Dutra (SMD) model [39]. Tanner and Milthorpe proposed a double viscosity model in function of a critical strain rate to describe the elastic behavior for low strain rates [88]. Papanastasiou [73] introduced an exponential regularization of the viscosity

$\mu(\dot{\gamma})=k \dot{\gamma}^{n-1}+\frac{\tau_{y}}{\dot{\gamma}}\left(1-e^{-m \dot{\gamma}}\right)$ 


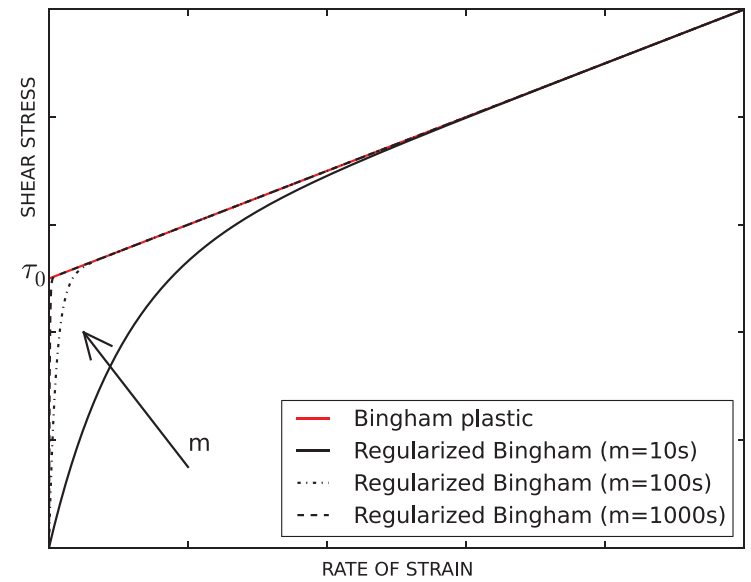

(a) Bingham model

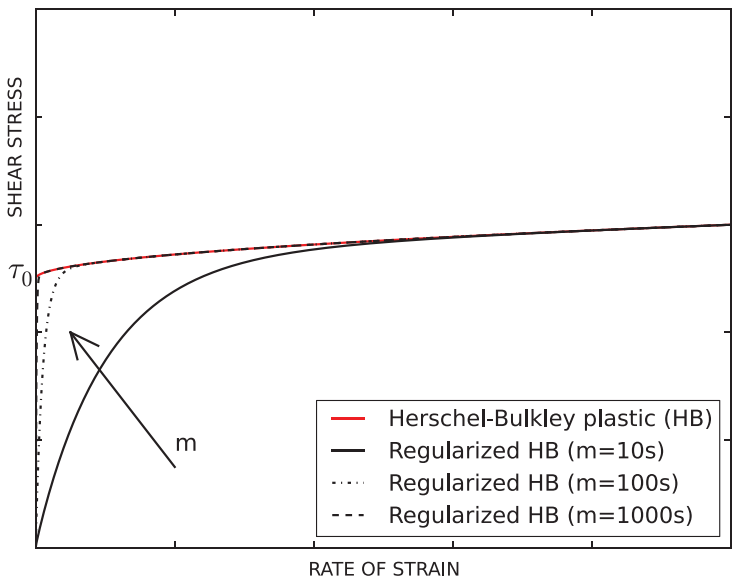

(b) Herschel-Bulkley model

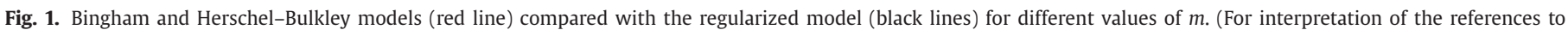
color in this figure legend, the reader is referred to the web version of this article).

where $m$ is a regularization parameter. When the rate of strain tends to zero $(\dot{\gamma} \rightarrow 0)$ the viscosity depends on the flow parameter $n$ : if $n>1$, the $\lim _{\dot{\gamma} \rightarrow 0} \mu(\dot{\gamma})=m \tau_{y}$ and, if $n=1$, the $\lim _{\dot{\gamma} \rightarrow 0} \mu(\dot{\gamma})=\mu+m \tau_{y}$; but if $n<1$, the $\lim _{\dot{\gamma} \rightarrow 0} \mu(\dot{\gamma})=\infty$. This means that for pseudoplastic fluids the viscosity is unbounded and a truncation procedure is needed. The regularization proposed by Souza-Mendez-Dutra solves this drawback applying the regularization to all the terms of the viscosity so that $\lim _{\dot{\gamma} \rightarrow 0} \mu(\dot{\gamma})=m \tau_{y}$ for any value of $n$ [39].

When $n=1$ the Bingham model is recovered, and the consistency index is equal to the plastic viscosity $\left(k=\mu_{0}\right)$.

The Bingham model also presents the singularity due to the perfectly rigid behavior below the yield stress. For Bingham plastics, Eq. (4), becomes

$\mu(\dot{\gamma})=\mu_{0}+\frac{\tau_{y}}{\dot{\gamma}}\left(1-e^{-m \dot{\gamma}}\right)$.

Fig. 1 shows the comparison between the Herschel-Bulkley and Bingham ideal models and the regularized ones of Eqs. (4) and (5), respectively.

The regularization proposed by Papanastasiou is used in the current work.

\section{Governing equations}

The problem of incompressible isothermal flow is defined by the Cauchy's equation of motion and the incompressibility constraint

$\rho\left(\partial_{t} \mathbf{u}+\mathbf{u} \cdot \nabla \mathbf{u}\right)-\nabla \cdot \sigma=\mathbf{f}$ in $\Omega, t \in[0, T]$

$\nabla \cdot \mathbf{u}=0$ in $\Omega$

$$
t \in[0, T]
$$

where $\Omega \subset \mathbb{R}^{d}$ ( $d$ is the space dimension) is the domain in a time interval $[0, T], \rho$ is the density of the fluid, and $\mathbf{f}$ are the volumetric forces. The stress tensor is decomposed as $\sigma=-p \mathbf{I}+\boldsymbol{\tau}$, where $p$ is the pressure and $\mathbf{I}$ is the identity tensor and $\boldsymbol{\tau}$ is the deviatoric stress tensor. Therefore, $\nabla \cdot \sigma=-\nabla p+\nabla \cdot \boldsymbol{\tau}$. If the regularized Bingham or Harschel-Bulkley model are used then

$\boldsymbol{\tau}=2 \mu(\dot{\gamma}) \boldsymbol{\varepsilon}(\mathbf{u})$ with $\mu(\dot{\gamma})$ defined by Eq. (4)) or Eq. (5), respectively. The problem is fully defined with the boundary conditions:

$\mathbf{u}(\mathbf{x}, t)=\overline{\mathbf{u}}(\mathbf{x}, t) \quad$ on $\quad \partial \Omega_{D}, \quad t \in[0, T]$,

$\mathbf{n} \cdot \boldsymbol{\sigma}(\mathbf{x}, t)=\mathbf{t}(\mathbf{x}, t) \quad$ on $\partial \Omega_{N}, \quad t \in[0, T]$,

where $\partial \Omega_{D}$ and $\partial \Omega_{N}$ are the Dirichlet and the Neumann boundaries, respectively $\left(\partial \Omega_{D} \cap \partial \Omega_{N}=\emptyset, \partial \Omega_{D} \cup \partial \Omega_{N}=\partial \Omega\right)$.

Steady-state flows are modeled by dropping the time derivative term in Eq. (6a). Likewise, the convective term can be neglected for low Reynolds numbers, as it is usually the case for viscoplastic fluids.

\section{Discrete model}

The governing equations (Eqs. (6)) are solved using mixed stabilized linear/linear finite elements for the spatial discretization.

The weak form of the problem is obtained using a Galerkin technique and the nonlinear terms of the momentum equation (i.e. the convective and viscous terms of Eq. (6a)) are linearized using a secant Picard method. The velocity $\mathbf{u}$ needs to belong to the velocity space $\mathcal{V} \in\left[\mathbf{H}^{1}(\Omega)\right]^{d}$ of vector functions whose components and their first derivatives are square-integrable and the pressure $p$ belongs to the pressure space $\mathcal{Q} \in \mathbf{L}_{2}$ of square-integrable functions.

Let $\mathcal{V}_{h} \subset \mathcal{V}$ be a finite element space to approximate $\mathcal{V}$, and $\mathcal{Q}_{h} \subset \mathcal{Q}$ a finite element approximation to $\mathcal{Q}$. Let $\Omega \subset \mathbb{R}^{d}$ be the domain in a time interval $[0, T]$, and $\Omega^{e}$ the elemental domain such that $\cup \Omega^{e}=\Omega$, with $e=1,2, \ldots, n_{e l}$ where $n_{e l}$ is the number of elements.

Therefore, the standard Galerkin discrete problem is finding $\mathbf{u}_{h} \in \mathcal{V}_{h}$ and $p_{h} \in \mathcal{Q}_{h}$ such that

$$
\begin{aligned}
& \int_{\Omega}\left[\rho \partial_{t} \mathbf{u}_{h} \cdot \mathbf{v}_{h}+\rho\left(\mathbf{u}_{h} \cdot \nabla \mathbf{u}_{h}\right) \cdot \mathbf{v}_{h} \quad+2 \mu(\dot{\gamma}) \nabla^{s} \mathbf{u}_{h}: \nabla^{s} \mathbf{v}_{h}\right. \\
& \left.-p_{h} \nabla \cdot \mathbf{v}_{h}-\mathbf{f}_{h} \cdot \mathbf{v}_{h}\right] d \Omega=0 \quad \forall \mathbf{v}_{h} \in \mathcal{V}_{h} \\
& \int_{\Omega}\left[q_{h} \nabla \cdot \mathbf{u}_{h}\right] d \Omega=0 \quad \forall q_{h} \in \mathcal{Q}_{h}
\end{aligned}
$$

$\mathbf{v}_{h}$ and $q_{h}$ are the velocity and the pressure weight functions belonging to velocity and pressure spaces, respectively, and some of the terms have been integrated by parts.

\subsection{Stabilized model}

In this work, low-order simplicial elements are used with the same linear interpolation for the velocity and pressure fields. This 
implies that the Ladyzenskaja-Babusłla-Brezzi condition, also called the inf-sup condition, is not respected and a stabilization technique is needed to overcome the instability of the pressure that may compromise the solution.

The stabilization employed is based on the subgrid scale approach proposed by Hughes ([14,48,50]). This proposes to split the velocity field $(\mathbf{u})$ into a part that can be represented by the finite element mesh $\left(\mathbf{u}_{h}\right)$ and another part that accounts for the unresolvable scale $(\overline{\mathbf{u}})$, that is, for the variation of the velocity that cannot be captured by the finite element mesh. This corresponds to a splitting of the space $\mathcal{V}$ into the space of the finite elements $\left(\mathcal{V}_{h}\right)$ and the subgrid space $(\overline{\mathcal{V}})$, so that $\mathcal{V}=\mathcal{V}_{h} \oplus \overline{\mathcal{V}}$.

The sub-scale $\overline{\mathbf{u}}$ is approximated from the residual of the momentum equation and it is evaluated inside each element, assuming the sub-scale to vanish on the boundary of each element. Different approximations of the sub-scale $\overline{\mathbf{u}}$ define different stabilization techniques.

In the present work, the Orthogonal Sub-grid Scale stabilization technique is used. This method was proposed by Codina ([31-33]) as a modification of the Algebraic Sub-Grid Scale (ASGS). In ASGS the sub-scale is taken proportional to the residual $\left(\mathbf{R}_{h}=\right.$ $\left.-\rho\left(\mathbf{u}_{h} \cdot \nabla \mathbf{u}_{h}\right)+\nabla \cdot \boldsymbol{\sigma}_{h}+\mathbf{f}_{h}\right)$ of the momentum equation, so that $\overline{\mathbf{u}}=-\tau_{1} \mathbf{R}_{h}$, where $\tau_{1}$ is a stabilization parameter. An application of ASGS to non-Newtonian fluid models can be found in [57] and [82]. Contrariwise, in the OSS the sub-scale is taken proportional to the orthogonal projection of the residual onto the finite element space

$\overline{\mathbf{u}}=-\tau_{1} P_{h}^{\perp}\left(\mathbf{R}_{h}\right)=-\tau_{1}\left(\mathbf{R}_{h}-P_{h}\left(\mathbf{R}_{h}\right)\right)$

where $P_{h}(\bullet)$ is the projection on the finite element space and $P_{h}^{\perp}(\bullet)=I(\bullet)-P_{h}(\bullet)$ is the orthogonal projection.

Residual based stabilization techniques such as ASGS and OSS do not introduce any consistency error, as the exact solution annuls the added terms, so that the stabilized model converges to the solution of the problem in continuum format. Also, if designed properly, the convergence rate is not altered; that is, the subscale terms must be appropriately dependent on the mesh size.

Constructing the subscale in the subspace orthogonal to the finite element subspace has several advantages over the many other possibilities. The main one is that it guarantees minimal numerical dissipation on the discrete solution, because it adds nothing to those components of the residual already belonging to the FE subspace. This maximizes accuracy for a given mesh, an issue always important and no less in nonlinear problems.

Additionally, in transient problems, the term corresponding to the time derivative belongs to the finite element space, and therefore, its orthogonal projection is null. This means that the mass matrix remains unaltered by the stabilization method, maintaining its structure and symmetry.

Moreover, if the residual can be split in two or more terms, e.g. if the stress tensor is split into its volumetric and deviatoric parts or if the residual includes a convective term, then the "cross products" in the stabilization terms can be neglected. This has three advantages: (i) it reduces the computational stencil, (ii) more selective norms can be defined for stability control and (iii) it has proved importance in problems involving singular or quasi-singular points both in linear and nonlinear problems.

The part of the residual to be orthogonally projected can be appropriately selected. For instance, in incompressible problems, only the gradient of the pressure needs to be added to ensure control of the pressure, with minimal numerical dissipation. These variants of the OSS, that can be considered to belong to the family of term-byterm stabilization methods, introduce consistency errors, but they are of optimal order and the final convergence rate of the scheme is not altered.
The discretized linearized problem, stabilized with OSS is, find $\mathbf{u}_{h}^{n+1}$ and $p_{h}^{n+1}$ such that

$$
\begin{aligned}
\int_{\Omega} & {\left[\frac{\rho}{\delta t}\left(\mathbf{u}_{h}^{n+1}-\mathbf{u}_{h}^{n}\right) \cdot \mathbf{v}_{h}+\rho\left(\mathbf{u}_{h}^{n+1} \cdot \nabla \mathbf{u}_{h}^{n+1, i}\right) \cdot \mathbf{v}_{h}\right.} \\
& \left.+2 \mu(\dot{\gamma})^{n+1, i} \nabla^{s} \mathbf{u}_{h}^{n+1}: \nabla^{s} \mathbf{v}_{h}-p_{h}^{n+1} \nabla \cdot \mathbf{v}_{h}-\mathbf{f}_{h}^{n+1} \cdot \mathbf{v}_{h}\right] d \Omega \\
& +\sum_{e} \int_{\Omega^{e}} \tau_{1} \rho\left(\mathbf{u}_{h}^{n+1} \cdot \nabla \mathbf{v}_{h}\right) \\
& \cdot\left[\rho \mathbf{u}_{h}^{n+1} \cdot \nabla \mathbf{u}_{h}^{n+1}+\nabla p_{h}^{n+1}-\mathbf{f}_{h}^{n+1}-\rho \mathbf{v}_{h}^{*} \cdot \mathbf{y}_{h}^{n+1}\right] d \Omega=0 \forall \mathbf{v}_{h} \in \mathcal{V}_{h}
\end{aligned}
$$

$$
\begin{aligned}
& \int_{\Omega}\left[q_{h} \nabla \cdot \mathbf{u}_{h}^{n+1}\right] d \Omega+\sum_{e} \int_{\Omega^{e}} \tau_{1} \nabla q_{h} \\
& \cdot\left[\left(\rho \mathbf{u}_{h}^{n+1} \cdot \nabla \mathbf{u}_{h}^{n+1}+\nabla p_{h}^{n+1}-\mathbf{f}_{h}^{n+1}\right)-\rho \mathbf{v}_{h}^{*} \cdot \mathbf{y}_{h}^{n+1}\right] d \Omega \\
& \quad=0 \forall q_{h} \in \mathcal{Q}_{h}
\end{aligned}
$$

where $\mathbf{y}_{\mathbf{h}}$ is the nodal projection defined as

$\mathbf{y}_{\mathbf{h}}{ }^{n+1}=P_{h}\left(\mathbf{u}_{h}^{n+1} \cdot \nabla \mathbf{u}_{h}^{n+1}+\frac{1}{\rho}\left(\nabla p_{h}^{n+1}-\mathbf{f}_{h}^{n+1}\right)\right)$

In compact notation, the projection of Eq. (12) is the solution of

$\left(\mathbf{y}_{\mathbf{h}}{ }^{n+1}, \mathbf{v}_{h}^{*}\right)=\left(\mathbf{u}_{h}^{n+1} \cdot \nabla \mathbf{u}_{h}^{n+1}+\frac{1}{\rho}\left(\nabla p_{h}^{n+1}-\mathbf{f}_{h}^{n+1}\right), \mathbf{v}_{h}^{*}\right)$

for all $\mathbf{v}_{h}^{*} \in \mathbf{V}_{h}^{*}$, being $\mathbf{V}_{h}^{*}$ equal to $\mathbf{V}_{h}$ extended with the vectors of continuous functions associated to the boundary nodes.

The stabilization parameter $\tau_{1}$ in Eqs. (11a) and (11b) is defined so to obtain a stable numerical scheme and an optimal velocity of convergence. Consequently, $\tau_{1}$ is calculated for each element as [32]

$\tau_{1}=\left[c_{1} \frac{\mu}{h_{e}^{2}}+c_{2} \frac{\rho\left|\mathbf{u}^{e}\right|}{h_{e}}\right]^{-1}$

where $h$ is the characteristic length of the eth element and $\left|\mathbf{u}^{e}\right|$ is the norm of velocity in the element. $c_{1}$ and $c_{2}$ are two coefficients that in the present work are chosen as $c_{1}=4$ and $c_{2}=2$ [32].

\section{Matrix form}

The solution system (11) is rewritten in matrix form as

$$
\begin{aligned}
& \mathbf{M} \frac{1}{\delta t} \mathbf{U}^{n+1}+\mathbf{K}\left(\mathbf{U}^{n+1}\right) \mathbf{U}^{n+1}+\mathbf{G} \mathbf{P}^{n+1} \\
& \quad+\mathbf{S}_{u}\left(\tau_{1} ; \mathbf{U}^{n+1}\right) \mathbf{U}^{n+1}-\mathbf{S}_{y}\left(\tau_{1} ; \mathbf{U}^{n+1}\right) \mathbf{Y}^{n+1}=\mathbf{F}^{n+1} \\
& \mathbf{D} \mathbf{U}^{n+1}+\mathbf{S}_{p}\left(\tau_{1}\right) \mathbf{P}^{n+1}-\mathbf{S}_{z}\left(\mathbf{U}^{n+1}\right) \mathbf{Y}^{n+1}=0 \\
& \mathbf{C}\left(\mathbf{U}^{n+1}\right) \mathbf{U}^{n+1}+\mathbf{G}_{\pi} \mathbf{P}^{n+1}=0
\end{aligned}
$$

where $\mathbf{U}$ and $\mathbf{P}$ are the vectors of nodal velocities and pressures, respectively, $\mathbf{Y}$ is the vector of nodal projections and $\mathbf{F}$ is the vector of nodal forces.

Finally, the matrix operators of Eqs. (15)) are defined as

$\mathbf{M}_{i j}^{a b}=\left(N^{a}, \rho N^{b}\right) \delta_{i j}$

$\mathbf{K}\left(\mathbf{U}^{n+1}\right)_{i j}^{a b}=\left(N^{a}, \rho \mathbf{u}_{h}^{n+1} \cdot \nabla N^{b}\right) \delta_{i j}+\left(\nabla N^{a}, 2 \mu \nabla^{s} N^{b}\right) \delta_{i j}$

$\mathbf{G}_{i}^{a b}=\left(N^{a}, \partial_{i} N^{b}\right)$

$\mathbf{F}_{i}^{a}=\left(N^{a}, \mathbf{f}_{i}\right)$ 


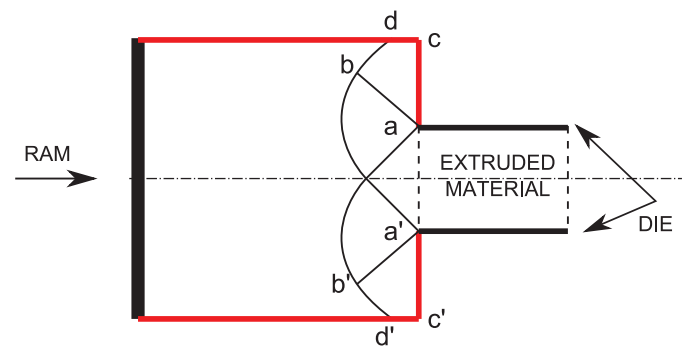

Fig. 2. Extrusion in a Bingham fluid. Slip lines according to Alexander [3].

$$
\begin{aligned}
& \mathbf{D}_{j}^{a b}=\left(N^{a}, \partial_{j} N^{b}\right) \\
& \mathbf{C}\left(\mathbf{U}^{n+1}\right)_{i j}^{a b}=\left(N^{a}, \mathbf{u}_{h}^{n+1} \cdot \nabla N^{b}\right) \delta_{i j} \\
& \mathbf{G}_{\pi}^{a b}=\left(N^{a}, \partial_{i} N^{b} / \rho\right)
\end{aligned}
$$

The stabilization operators of Eqs. (15) are

$\mathbf{S}_{\mathbf{u}}\left(\tau_{1}, \mathbf{U}^{n+1}\right)_{i j}^{a b}=\left(\tau_{1} \mathbf{u}_{h}^{n+1} \cdot \nabla N^{a}, \rho \mathbf{u}_{h}^{n+1} \cdot \nabla N^{b}\right) \delta_{i j}$

$\mathbf{S}_{\mathbf{y}}\left(\tau_{1} \mathbf{u}_{h}^{n+1}\right)_{i j}^{a b}=\left(\tau_{1} \mathbf{u}_{h}^{n+1} \cdot \nabla N^{a}, \rho N^{b}\right) \delta_{i j}$

$\mathbf{S}_{\mathbf{p}}\left(\tau_{1}\right)^{a b}=\left(\tau_{1} \mathbf{u}_{h}^{n+1} \cdot \nabla N^{a}, \nabla N^{b}\right)$

$\mathbf{S}_{\mathbf{z}}\left(\tau_{1}\right)_{j}^{a b}=\left(\tau_{1} \partial_{j} N^{a}, \rho N^{b},\right)$

\section{Numerical results: Bingham fluids}

\subsection{Extrusion}

\subsubsection{Description of the problem}

The first example is the extrusion process of a Bingham fluid. Extrusion is widely used in several industrial processes such as metal forming, manufacturing, food production, etc. Real applications are usually in three dimensions; nevertheless, a plane strain $2 D$ analysis provides very useful information on the evolution of the plastic region and gives an estimation of the forces required in the process.

The slip-lines theory was first introduced by Prandtl at the beginning of the XX century [77]. This methodology was originally used in plane strain problems to estimate the stress field and the related velocity field in perfect plastic materials with the Von Mises (or Tresca) yield criterion. The approach was generalized by Mandel [62], who introduced other yield criteria and analyzed the plane stress case [61].

The slip lines are tangent to the direction of the maximum tangential stress and are the trajectories of the maximum shear stress. In plain strain, the plastic flow coincides with the maximum shear stress direction. Therefore, rigid-plastic material "slips" in the direction of the maximum shear stress lines.

In 1948, Hill [47] used the slip line theory to analytically solve the problem of direct frictionless extrusion in a die with a $50 \%$ reduction of its section. In 1961, Alexander [3] demonstrated that if the reduction of the die section is of $2 / 3$, there exists a part of the yielded region (the area ABDC in Fig. 2) in which the average pressure coincides with the extrusion pressure $p$

$p=\frac{4}{3}\left(1+\frac{\pi}{2}\right) \tau_{y}$

where $\tau_{y}$ is the yield stress.

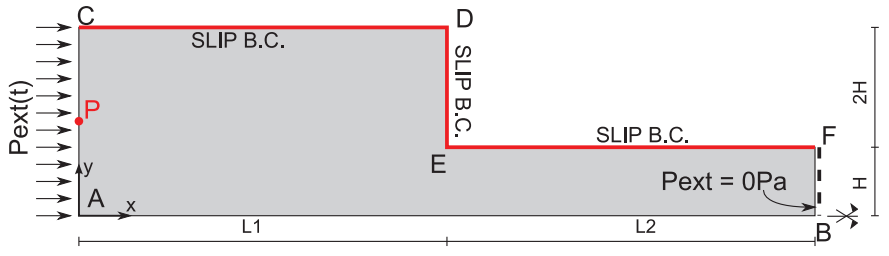

Fig. 3. Extrusion in a Bingham fluid. Geometry and boundary conditions.

The extrusion process can be numerically simulated using either a Lagrangian plastic flow or an elasto-plastic solid. In the first case, the elastic deformation is neglected and the material follows the Von Mises yield criterion and an associated flow rule. Zienkiewicz [93] and Oñate [72] applied this approach to analyse the plain stress problem without hardening using a Lagrangian mesh moving with the material. In the second case, the elastic strains are considered, which complicates the problem introducing both geometrical and material non linearities. In 1984, Lee [59] published one of the first examples of an extrusion problem using a large deformation elasto-plastic approach. He used an updated Lagrangian technique and the Von Mises yield criterion with hardening.

A widely used alternative is to use an Eulerian viscoplastic flow and a Von Mises yield criterion $([38,45,55,74])$. In this case, the material follows a rigid-plastic law with a very low plastic viscosity (almost perfect plasticity). Once the yield stress is reached, a high localization of the strain rate occurs. This can be identified with the slip lines of Prandtl theory. This is the formulation used in this work with the objective of identifying the yielded and unyielded regions, the evolution of the stream lines and of the slip lines. The calculated pressure on the ram is compared with the analytical solution given by Eq. (18).

\subsubsection{Model and results}

The geometry and boundary conditions used are presented in Fig. 3. A reduction of $2 / 3$ of the cross section is considered. A slip condition is imposed on the wall boundaries CDEF and C'D'E'F', no wall laws are considered. This means that on CD and EF $u_{y}=0$ while $u_{x}$ is left free and on DE $u_{x}=0$ while $u_{y}$ is left free. Symmetry conditions are imposed on $\mathrm{AB}\left(u_{y}=0\right)$. An increasing normal stress is imposed on $\mathrm{CC}^{\prime}$. This represents the ram pressure that increases linearly with time from $p=0 \mathrm{~Pa}$ at the initial time $(t=0$ s) to $p=5000 \mathrm{~Pa}$ at $t=1 \mathrm{~s}$. The vertical component of velocity is set to zero on CC'. The pressure is set to zero in point $\mathrm{B}$, and the horizontal velocity is left free in point $\mathrm{E}$.

A $2 D$ plane strain simulation is carried on. Exploiting the symmetry of the problem, only half of the domain is discretized using 2821 nodes 5340 and linear/linear $(P 1 / P 1)$ triangular elements (see Fig. 4).

The material parameters are summarized in Table 1 where the regularization coefficient employed for the Bingham model is also given.

The example is solved as a series of steady-state problems with increasing ram pressure. Two scenarios have been taken into account: with and without the convective term in the momentum equation. Fig. 5 shows the velocity evolution on point $\mathrm{P}$ while the pressure on the ram is increased, in comparison with the analytical solution (continuous line). At $t=0.69 \mathrm{~s}$ the flow is fully developed and the yielded regions are completely defined. The numerical pressure for yielding is $P_{\text {num }}=3400 \mathrm{~Pa}$, while the analytical solution is $P_{a n}=3428 \mathrm{~Pa}$ according to Eq. (18).

If the convection term is included in the momentum equation (black dotted line in Fig. 5), it is necessary to increase the external pressure in order to overcome the inertial effects once the yield stress is achieved. This does not happen when the convective term 


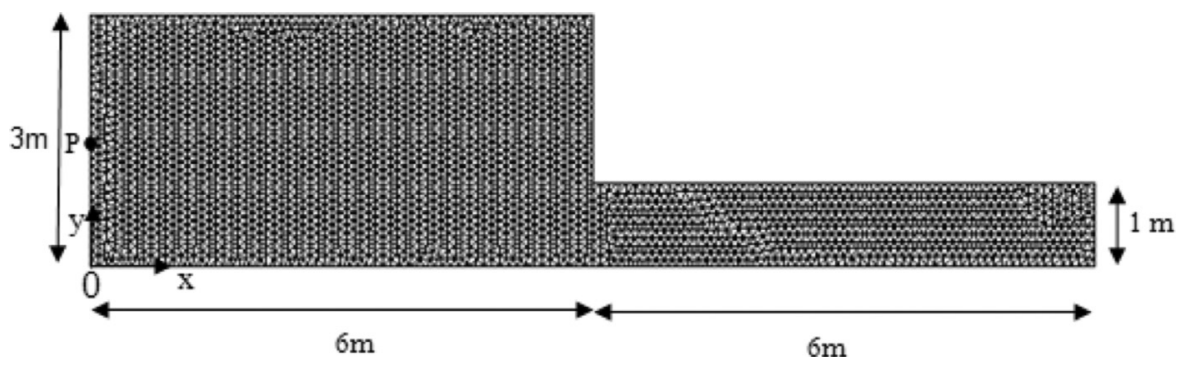

Fig. 4. Extrusion in a Bingham fluid. Mesh used in the calculation: 2821 nodes and 5340 linear triangular elements.

Table 1

Extrusion in a Bingham fluid. Material parameters and regularization coefficient.

\begin{tabular}{lll}
\hline Material properties & & \\
\hline Plastic viscosity & $\mu_{0}$ & $10^{-6} \mathrm{~Pa} \cdot \mathrm{s}$ \\
Density & $\rho$ & $100 \mathrm{~kg} / \mathrm{m}^{3}$ \\
Yield stress & $\tau_{y}$ & $1000 \mathrm{~Pa}$ \\
$\begin{array}{l}\text { Regularization } \\
\text { Regularization coefficient }\end{array}$ & $m$ & $1000 \mathrm{~s}$ \\
\hline
\end{tabular}

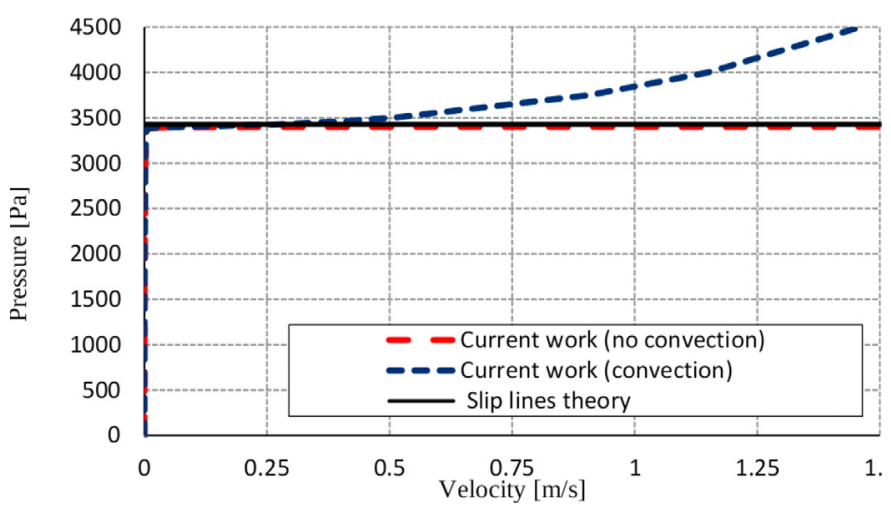

Fig. 5. Extrusion in a Bingham fluid. Pressure-velocity curve in point P (see Fig. 4). Comparison between the analytical solution and the numerical results. (For interpretation of the references to color in this figure text, the reader is referred to the web version of this article). is neglected (red dotted line in Fig. 5). In this case, once the slip lines have developed, very large velocities are achieved with a very small increment of external pressure.

Fig. 6 presents the stream lines evolution during the extrusion process. An abrupt change in the smoothness of the streamlines is observed when the slip lines appear (Fig. 6(c) and (d)). Fig. 6 also shows the yielded (dark) and unyielded (fair) regions above and below the critical strain rate $\left(\dot{\gamma}_{\text {crit }}=0.01688 \mathrm{~s}^{-1}\right.$, correspondent to $\left.\tau=\tau_{y}\right)$.

The evolution of the velocity field is presented in Fig. 7. It can be observed that while at $t=0.6 \mathrm{~s}$ almost all the domain is solid and just a very small region has reached the yield threshold, at $t=0.678 \mathrm{~s}$ the extrusion mechanism and the slip lines are fully developed. These lines coincide with the slip lines of the classical plastic theory [61].

\subsection{Flow around a cylinder between two parallel planes}

\subsubsection{Description of the problem}

The flow around a cylinder in a confined Bingham fluid is studied in this second example. The flow around an obstacle was initially studied considering a spherical object. This classical problem in computational fluid dynamics has several practical applications in different engineering fields: from segregation in food industry, to transport of mud in geotechnical engineering or aerosols in environmental engineering, etc. The general problem is the suspension of large particles in a fluid with a yield threshold. The falling or settlement of the particles can only occur if the gravity force exceeds the yield limit $([23,78,86])$.

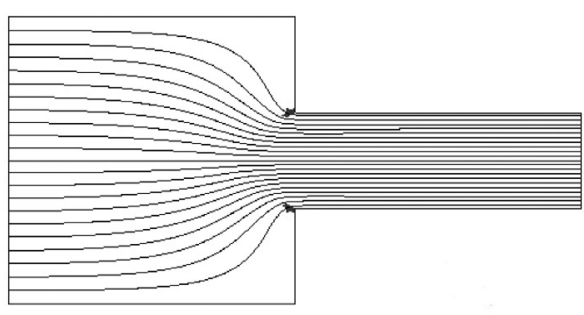

(a) $t=0.6 \mathrm{~s}$

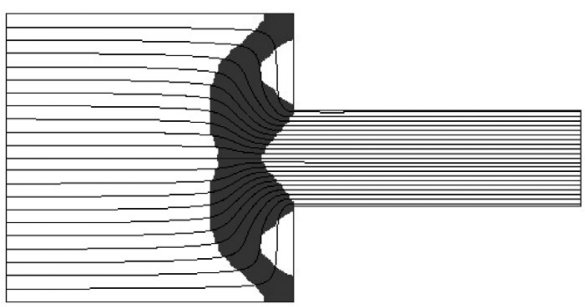

(c) $t=0.678 \mathrm{~s}$

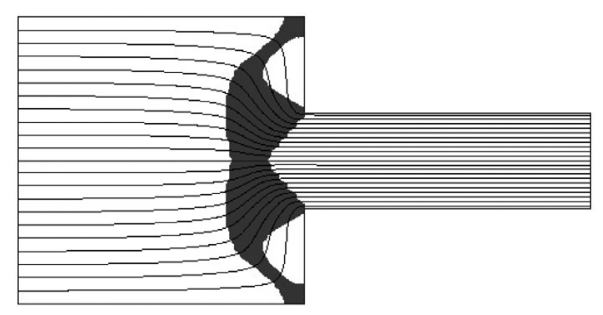

(b) $t=0.677 \mathrm{~s}$

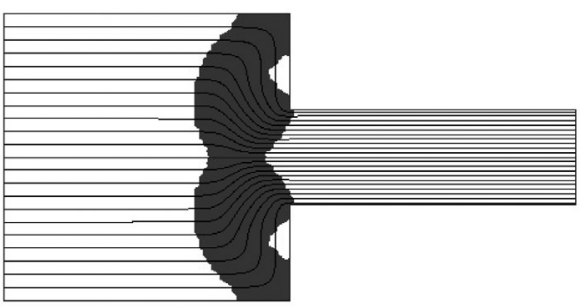

(d) $t=0.68 \mathrm{~s}$

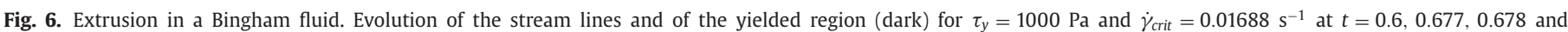
$0.68 \mathrm{~s}$. 


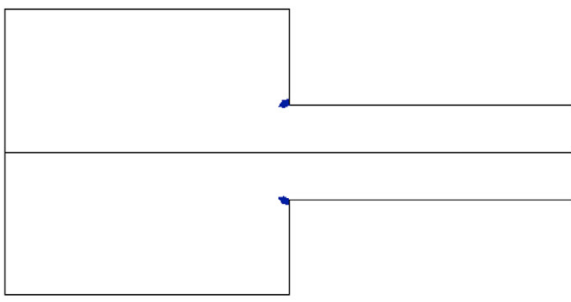

(a) $t=0.6 \mathrm{~s}$

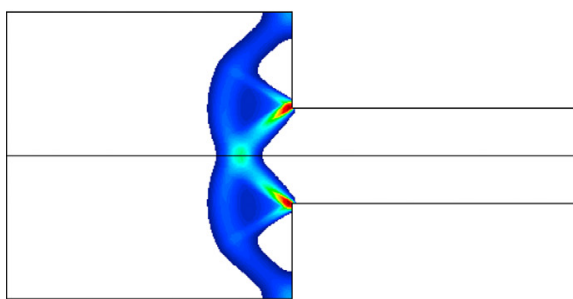

(c) $t=0.678 \mathrm{~s}$

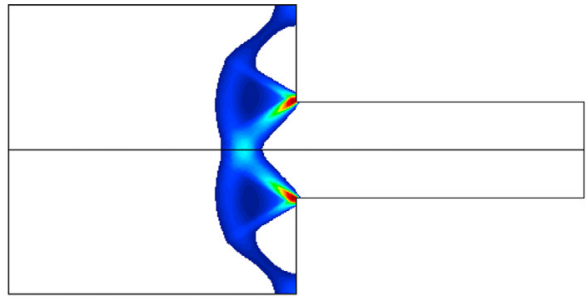

(b) $t=0.677 \mathrm{~s}$

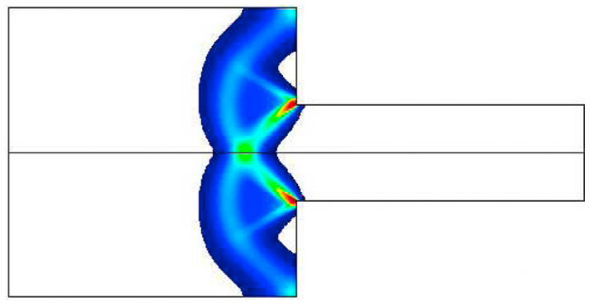

(d) $t=0.68 \mathrm{~s}$

Fig. 7. Extrusion in a Bingham fluid. Evolution of the velocity field for $\tau_{y}=1000 \mathrm{~Pa}$ and $\dot{\gamma}_{\text {crit }}=0.01688 \mathrm{~s}^{-1}$ at $t=0.6,0.677,0.678$ and $0.68 \mathrm{~s}$.

The viscoplastic flow around an obstacle has been widely studied both numerically and experimentally ([23,25,44,90]). For the specific case of Bingham plastics, many authors have proposed different solutions for the flow around a sphere subjected to gravity force between two parallel planes or in an infinite domain $([12,60,66,94])$. Moreover, Roquet and Sarmito [80] studied the effect of an additional pressure gradient and Slijecpflević and Perić [85] studied the movement of a sphere inside a cylinder.

Nowadays, there exists abundant literature on a sphere falling either in a pseudoplastic, viscoplastic or viscoelastic fluid for low Reynolds numbers [24]. Contrariwise, not many authors have treated the movement of a cylinder in a non-Newtonian fluid.

The aim of this example is to define the yielded zones and the hydrodynamic drag force in terms of the geometrical configuration of the parallel planes and the cylinder.

\subsubsection{Non-dimensional forces}

In this and the following examples a series of non-dimensional quantities will be used to present the results. These quantities are defined here.

Being $x$ the direction of the flow and $y$ its orthogonal direction in the plane (see Fig. 8(a)), the drag force $\left(F_{D}\right)$ and lift force $\left(F_{L}\right)$ acting on the cylinder can be calculated as

$F_{D}=l R \int_{0}^{2 \pi} t_{x} d \theta=4 l R \int_{0}^{\pi / 2}\left[\sigma_{x x} \cos \theta+\sigma_{x y} \sin \theta\right] d \theta$

and

$F_{L}=l R \int_{0}^{2 \pi} t_{y} d \theta=4 l R \int_{0}^{\pi / 2}\left[\sigma_{x y} \cos \theta+\sigma_{y y} \sin \theta\right] d \theta$

where $R=1 \mathrm{~m}$ is the radius and $l=1 \mathrm{~m}$ is the height of the cylinder. The traction vector $\mathbf{t}^{T}=\left(t_{x}, t_{y}\right)$ is defined by the stress components in the $x y$ plane (i.e., $\sigma_{x x}, \sigma_{y y}, \sigma_{x y}$ ) and of angle $\theta$ between the normal to the cylinder and the $x$ axis as $t_{x}=\sigma_{x x} \cos \theta+\sigma_{x y} \sin \theta$ and $t_{y}=\sigma_{x y} \cos \theta+\sigma_{y y} \sin \theta$.

The non-dimensional drag and lift coefficients in the specific case of a Bingham fluid are

$F_{D}^{*}=\frac{F_{D}}{\mu V l} ; \quad F_{L}^{*}=\frac{F_{L}}{\mu V l}$

An information on the relevance of the yield stress in the resistance that the flow provides to the movement of the cylinder

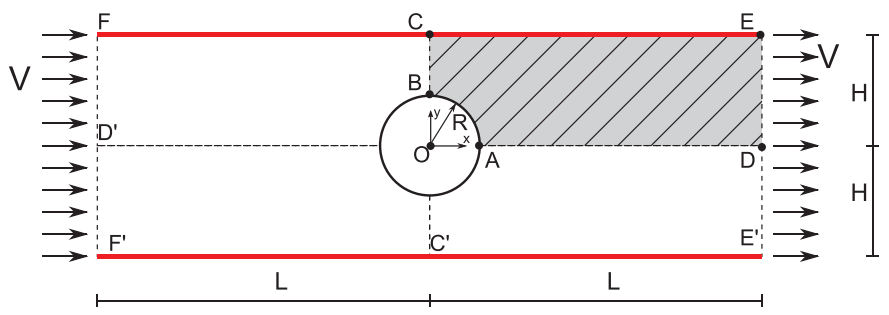

(a) Complete domain

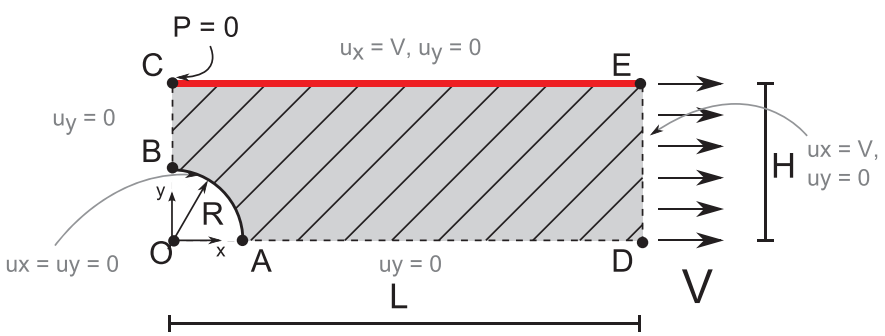

(b) Reduced domain used in the analysis

Fig. 8. Cylinder in a Bingham fluid. Geometry and boundary conditions.

is given by the drag coefficient $F_{D}^{\prime}$. This is by definition the ratio between the drag force and the yield stress

$F_{D}^{\prime}=\frac{F_{D}}{\tau_{y}}$

Finally, the last non-dimensional quantity used in the paper is the non-dimensional yield stress $\tau_{y}^{*}$ associated to the drag force

$\tau_{y}^{*}=\frac{2 \tau_{y} \pi R^{2}}{F_{D}}$.

\subsubsection{Model and results}

The cylinder with radius $R=1 \mathrm{~m}$ is located between two infinite parallel planes. The distance between the planes is $2 H$ and the center of the cylinder is at distance $H$ from both of them. The system of reference is attached to the center of the cylinder and it is considered fixed (Fig. 8(a)). The planes are moving with velocity $\mathrm{V}$ as well as the lateral sides of the computational domain, located 
Table 2

Cylinder in a Bingham fluid. Material parameters and regularization coefficient.

\begin{tabular}{|c|c|c|}
\hline Material properties & & \\
\hline Plastic viscosity & $\mu_{0}$ & $1 \mathrm{~Pa} \cdot \mathrm{s}$ \\
\hline Yield stress & $\tau_{y}$ & $0,0.05,0.5,5,50,500 \mathrm{~Pa}$ \\
\hline Bingham number & $B n$ & $0,0.1,1,10,100,1000$ \\
\hline $\begin{array}{l}\text { Regularization } \\
\text { Regularization coefficient }\end{array}$ & $m$ & $1000 \mathrm{~s}$ \\
\hline
\end{tabular}

Table 3

Cylinder in a Bingham fluid. Domains and meshes considered.

\begin{tabular}{lrrrr}
\hline Case & H : $\mathbf{R}$ & \multicolumn{1}{c}{ L : $\mathbf{R}$} & Nodes & Elements \\
\hline M1 & $2: 1$ & $12: 1$ & 783 & 1401 \\
M2 & $4: 1$ & $24: 1$ & 3494 & 6623 \\
M3 & $10: 1$ & $60: 1$ & 5371 & 10,245 \\
M4 & $50: 1$ & $250: 1$ & 13513 & 25,473 \\
\hline
\end{tabular}

sufficiently far from the cylinder. No slip is assumed on the surface of the cylinder and inertial effects are ignored $(R e \approx 0)$. The flow has double symmetry, with respect both to the vertical and to the horizontal axes. For this reason, just a quarter of the domain is analyzed (see Fig. 8(b)) $[8,75]$ ).

Fig. 8(b) shows a schematic description of the boundary conditions used. A no slip condition is applied on line $A B$, orthogonal velocity and tangential stresses are zero on lines $\mathrm{BC}$ and $\mathrm{AD}$. The velocity is fixed on ED and on the upper wall where the vertical component $u_{y}=0$ and the horizontal one $u_{x}=V=1 \mathrm{~m} / \mathrm{s}$. Pressure is set to zero on $C$ to determine univocally the pressure field. The length of the domain ( $L$ in Fig. $8($ b) $)$ is sufficiently large to ensure that the flow is completely developed.

The properties of the material are summarized in Table 2. The Bingham number $(\mathrm{Bn})$ in Table 2 is a non-dimensional quantity representing the ratio between the yield and the viscous stresses and it is calculated as $B n=\tau_{y}(2 R) / \mu_{0} V$ where $\tau_{y}$ is the yield stress, $H$ is the radius of the die, $\mu_{0}$ is the plastic viscosity and $V$ is the velocity of the fluid. A range of yield stresses (and, therefore, of Bingham numbers) is taken into account.

Different relations $H: R$ and $L: R$ have been considered to assess the effect of the domain size on the results. These are summarized in Table 3. In all the cases a more refined mesh is considered close to the cylinder (see Fig. 9).

The results obtained in terms of yielded regions, drag force and stream lines are coherent with those obtained by Mitsoulis [66]. In Fig. 10 the yielded and unyielded regions are shown for different Bingham numbers for two different geometrical ratios $H: R=4: 1$ and $H: R=10: 1$. Fig. 10(a) and (f) show the streamlines in the Newtonian case (i.e., $B n=0$ ). In the first case, the larger relative dimension of the cylinder leads to a steeper gradient of velocity in the $y$ direction. For Bingham numbers $B n>10$, the drag force is independent from $H: R$. The yielded/unyielded regions, the recir-

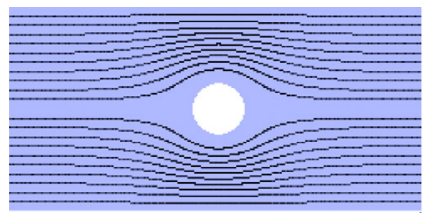

(a) $B n=0, H: R=4: 1$

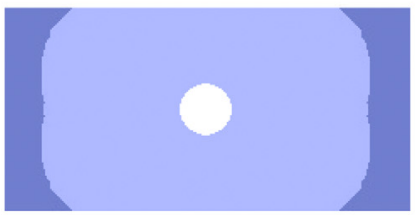

(b) $B n=0.1, H: R=4: 1$

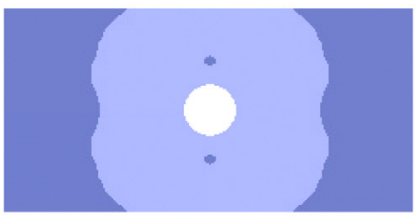

(c) $B n=1, H: R=4: 1$

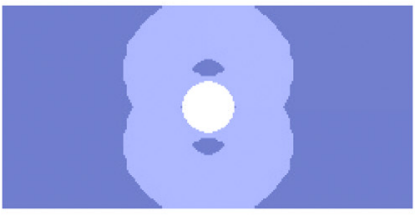

(d) $B n=10, H: R=4: 1$

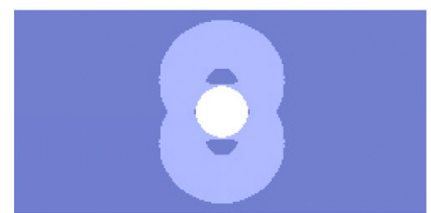

(e) $B n=100 s, H: R=4: 1$

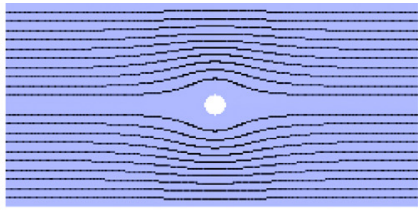

(f) $B n=0, H: R=10: 1$

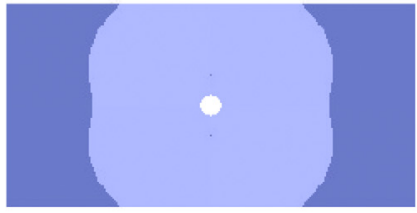

(g) $B n=0.1, H: R=10: 1$

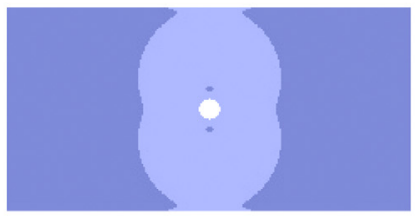

(h) $B n=1, H: R=10: 1$

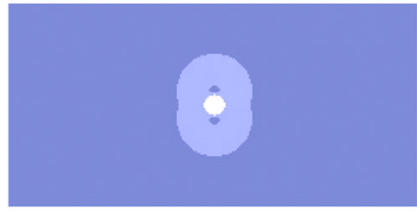

(i) $B n=10, H: R=10: 1$

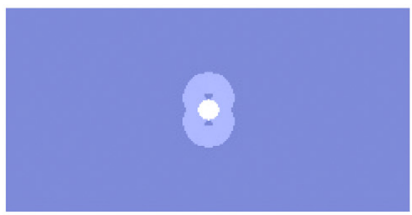

(j) $B n=100 \mathrm{~s}, H: R=10: 1$
Fig. 10. Cylinder in a Bingham fluid. Stream lines and yielded (fair) and unyielded (dark) region for different Bingham numbers. On the left $H: R=4: 1$, on the right $H: R=10: 1$

culation and stagnation regions appear similarly to what happens in the case of a sphere. It is worth observing that as $B n$ increases:

- The yielded region around the cylinder decreases

- The unyielded region surrounds the cylinder. This process is more evident in the case $H: R=10: 1$, confirming that the wall effect is not negligible in the case $H: R=4: 1$.

- The recirculation islands immersed in the yielded region appear and get closer to the cylinder in a symmetric way. They finally adhere to the cylinder for $B n=100$.

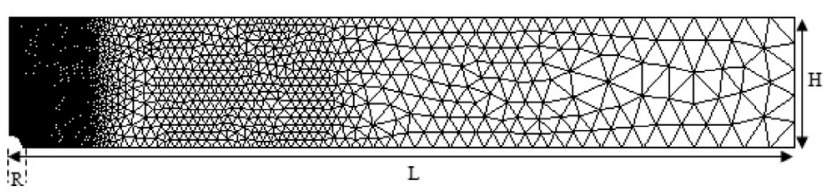

(a) Mesh of the whole domain

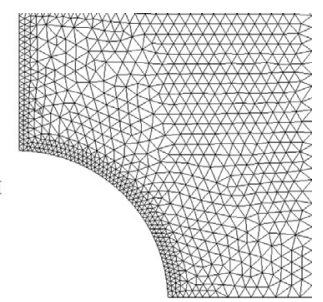

(b) Mesh around the cylinder

Fig. 9. Cylinder moving in a Bingham fluid. Unstructured mesh of case $M 3$ with $H: R=10, L: R=60$. 


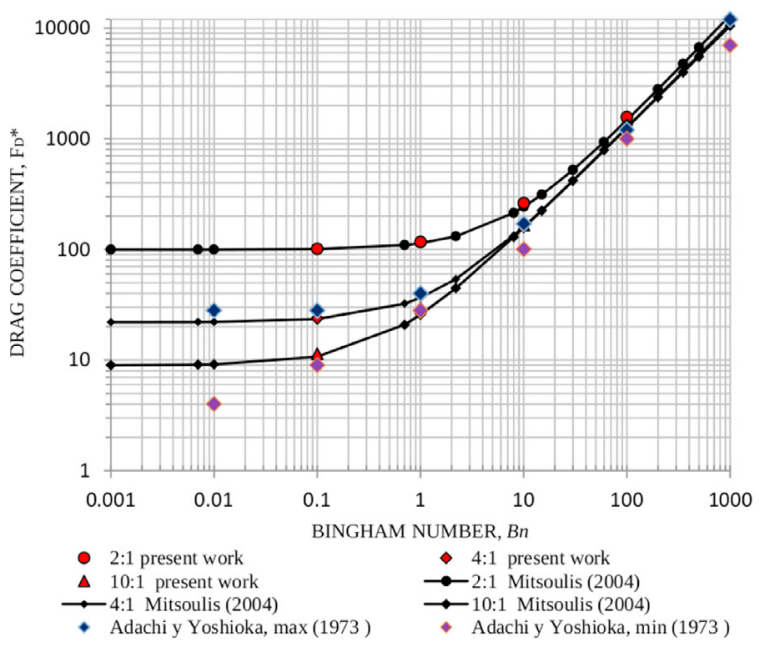

(a) Drag coefficient vs $B n$

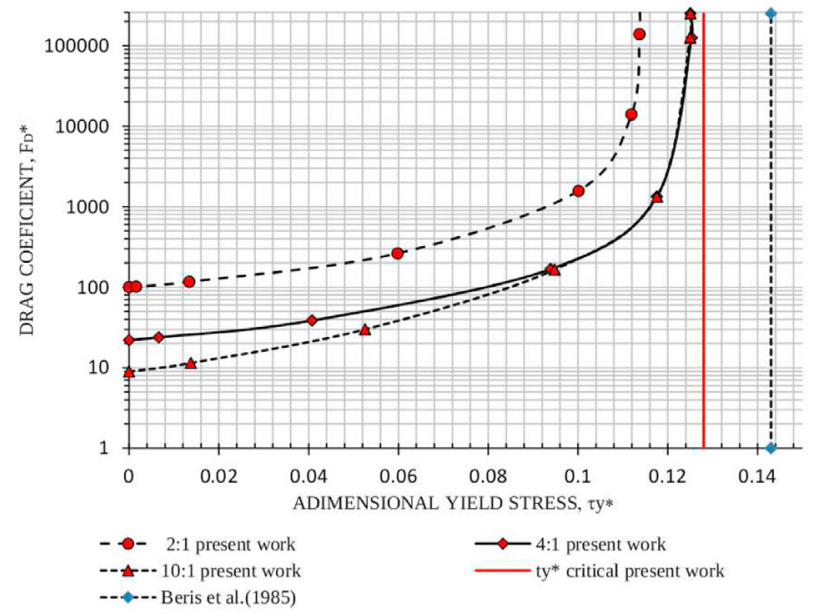

(b) Drag coefficient vs non-dimensional $\tau_{y}^{*}$

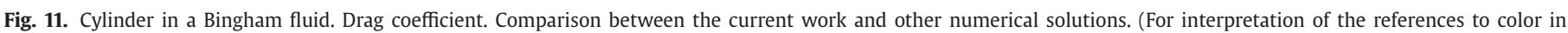
this figure in the text, the reader is referred to the web version of this article).

- The stagnation zone appears at the side of the cylinder.

- The stagnation zone get smaller than the recirculation one.

The dimension and shape of the polar caps appearing in the stagnation regions are similar to the results presented in [8] and [91].

There is little information on the drag coefficient of a cylinder moving in a viscoplastic fluid. Roquet and Saramito [80] and Mitsoulis [66] present some studies on this specific problem. In Fig. 11(a) the non-dimensional drag coefficient, Eq. (21), is plotted versus the Bingham number for the different cases analyzed and the results are compared with those of Mitsoulis showing a good agreement. It is worth observing that, as the Bingham number increases, the non-dimensional drag coefficient increases and becomes independent from the relation $H: R$ (for $H: R>2$ ). When $B n \rightarrow 0$, the non-dimensional drag reaches the value of the drag of a Newtonian fluid and when $B n \rightarrow \infty$ it tends to $F_{D}^{*}=1.14 B n$. This limit was also identified by Mitsoulis and Huigol [69]. The results obtained in this work are in the range of the limit values obtained by Adachi and Yoshioka [2] with their max and min theorem.

Fig. 11(b) shows that for high values of the non-dimensional yield stress the drag increases. The growth is progressively more steep as it gets to the critical limit of $\tau_{y}^{*}=0.128$ (the red vertical line of Fig. 11(b)). At this value of the yield stress, the drag force balances with the buoyancy force.

\section{Numerical results: Herschel-Bulkley fluids}

\subsection{Flow around a cylinder in an infinite medium}

\subsubsection{Description of the problem}

The problem treated in this section is similar to the one presented in Section 6.2, but now the medium is infinite the flow follows the Herschel-Bulkley model. This is a complex and seldom studied phenomenon. In the literature there exist some studies on a sphere moving in a tube filled with a Herschel-Bulkley fluid at $R e \approx 0$ ([5], [6]). Some experimental results were provided by Atapattu [4] and, more recently, some experiments were performed on the flow around several spheres at low $\operatorname{Re}(\operatorname{Re}<1)$ confirming the difficulties on managing very low velocities ([63], [86]). Some authors have studied the movement of cylinders of different sizes inside a tube [68] and the flow around objects with different

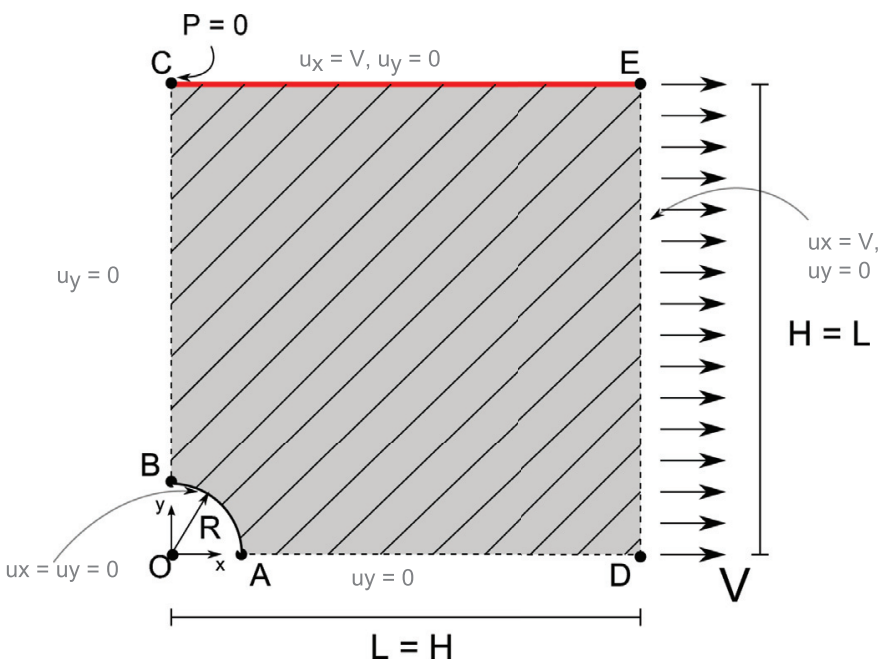

Fig. 12. Cylinder in an Herschel-Bulkley fluid. Geometry and boundary conditions.

shapes with $R e$ in the range $\left[10^{-1}-10^{-8}\right.$ ] [54]. Mitsoulis provided a review of the results obtained for different problems on Bingham and Herschel-Bulkley flows [67] where the flow around a sphere in a viscoplastic medium is mentioned.

The flow around a cylinder in a Herschel-Bulkley pseudoplastic fluid in an infinite domain was studied by De Besses [36]. Tanner [87] presents numerical results for a cylinder moving in a pseudoplastic fluid (governed by a power law, without yield threshold) in an infinite domain. The problem in a confined domain was studied by Missirlis et al. [65] and [84]. Barthi et al. [9] included also dilatant fluids $(0.6<n<2)$.

All the works mentioned are based on finite elements, except Bharti et al. [9], where finite volumes were employed, and Tanner and Milthorpe [88], who used boundary elements. Sivakumar [84] compared finite elements and finite volumes results demonstrating the equivalence of both approaches.

The case of non-inertial flow of a Newtonian fluid around a cylinder in an infinite domain has no analytical solution; the reason being related to the shape of the streamlines far away from the cylinder, what is known as the Stoke's paradox [89]. The 
Table 4

Cylinder in an Herschel-Bulkley fluid. Material parameters and regularization coefficient.

\begin{tabular}{|c|c|c|}
\hline \multicolumn{3}{|l|}{ Material properties } \\
\hline Yield stress & $\tau_{y}$ & $1,10,100 \mathrm{~Pa}$ \\
\hline Generalized Bingham number & $B n^{*}$ & $1,10,100$ \\
\hline Flow index & $n$ & $0.25,0.5,0.75,1,2$ \\
\hline $\begin{array}{l}\text { Regularization } \\
\text { Regularization coefficient }\end{array}$ & $m$ & $1000 \mathrm{~s}$ \\
\hline
\end{tabular}

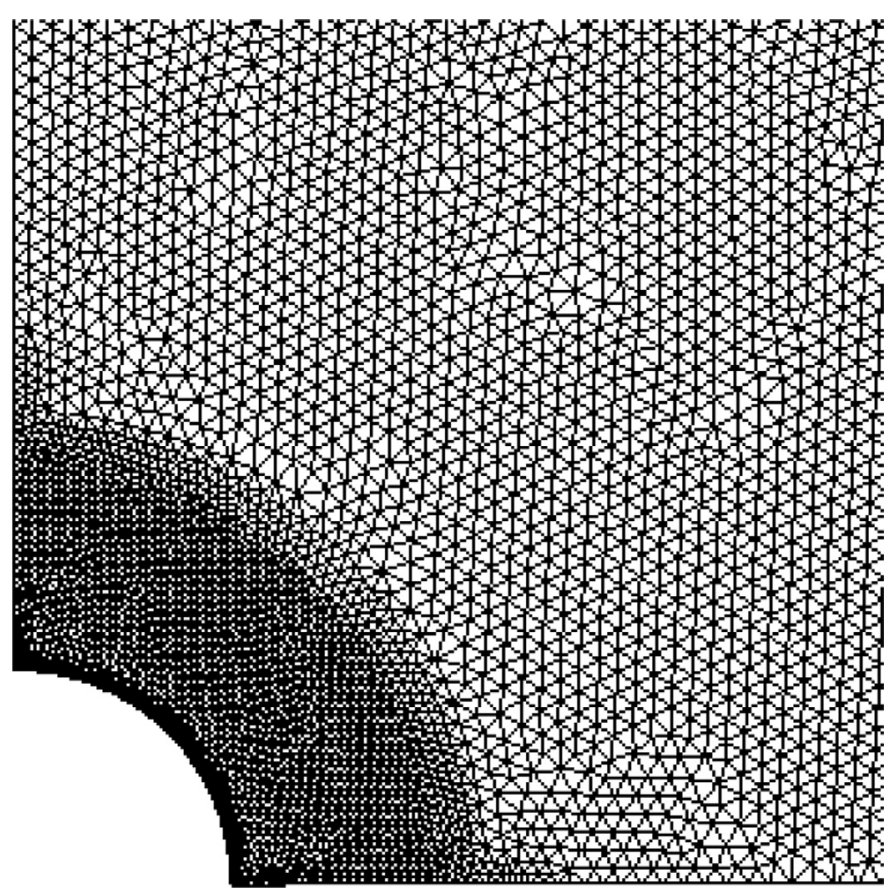

Fig. 13. Cylinder in an Herschel-Bulkley fluid. Unstructured mesh.

paradox does not present for pseudoplastic fluids $(n \leq 1)$ and it is still unclear if it is present or not for dilatant flows $(n>1)$.

In the case of a flow in a finite domain the analytical solution does exist for all values of the flow index $n$ ([26], [88]).

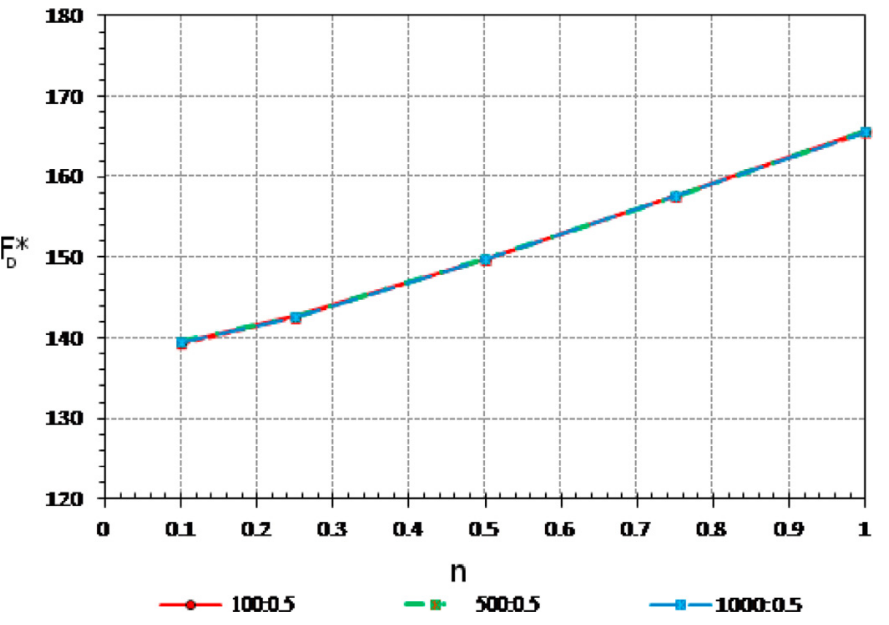

Fig. 14. Cylinder in an Herschel-Bulkley fluid. Drag force coefficient in terms of the relation $L: R$.

Table 5

Cylinder in an Herschel-Bulkley fluid. Domains and meshes considered.

\begin{tabular}{lrll}
\hline Case & \multicolumn{1}{l}{ L : R } & Nodes & Elements \\
\hline M1 & $100: 0.5$ & 9367 & 18351 \\
M2 & $500: 0.5$ & 9500 & 18601 \\
M3 & $1000: 0.5$ & 9571 & 18729 \\
\hline
\end{tabular}

The objective of the current work is to study the flow around a cylinder in an infinite Herschel-Bulkley fluid domain. The determination of the drag force, the yielded and unyielded zones, as well as the recirculation and stagnation zones is carried out for different generalized Bingham numbers. The generalized Bingham number for an Herschel-Bulkley fluid is defined as $B n^{*}=\frac{\tau_{y}}{k}\left(\frac{H}{V}\right)^{n}$.

Non inertial $R e \approx 0$ is assumed in all the examples.

\subsubsection{Non-dimensional forces}

The non-dimensional drag and lift coefficients in the specific case of a Herschel-Bulkley fluid are defined as

$F_{D}^{*}=\frac{\frac{F_{D}}{R l}}{k\left(\frac{V}{R}\right)^{n}}=\frac{F_{D}}{k R^{1-n} V^{n} l} ; \quad F_{L}^{*}=\frac{\frac{F_{L}}{R l}}{k\left(\frac{V}{R}\right)^{n}}=\frac{F_{L}}{k R^{1-n} V^{n} l}$

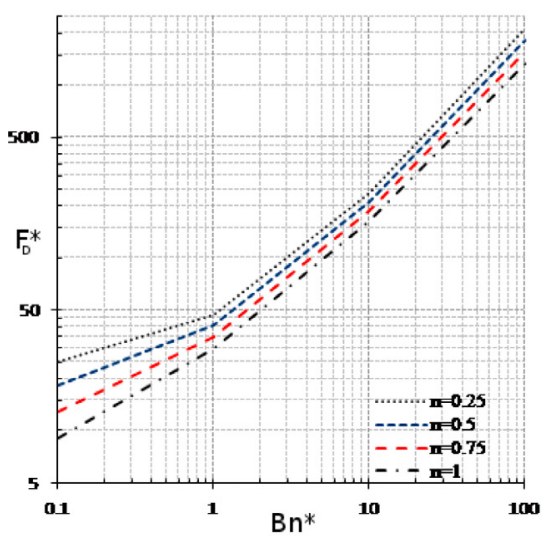

(a) Drag force vs $B n$

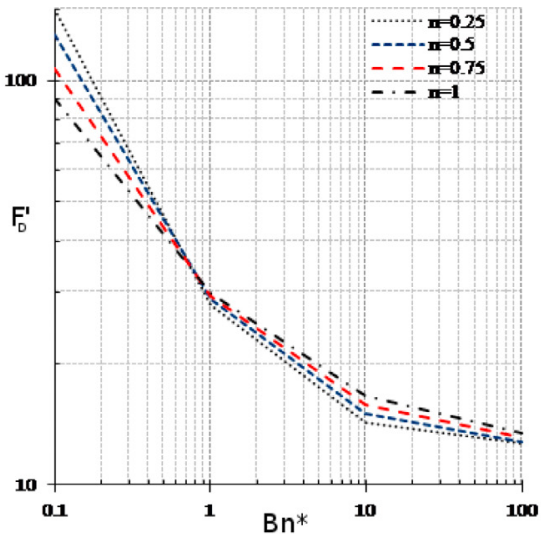

(b) Drag coefficient related to the yield stress

Fig. 15. Cylinder in an Herschel-Bulkley fluid. Drag force and Drag coefficient for different flow indexes $n$. 


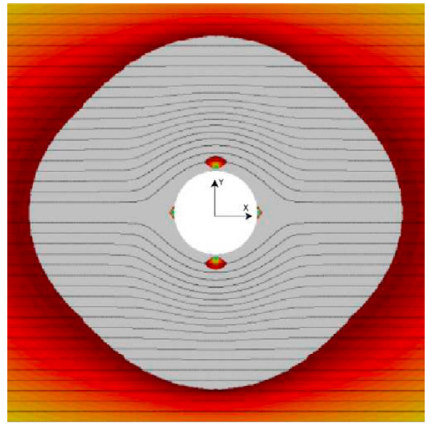

(a) $B n^{*}=10$

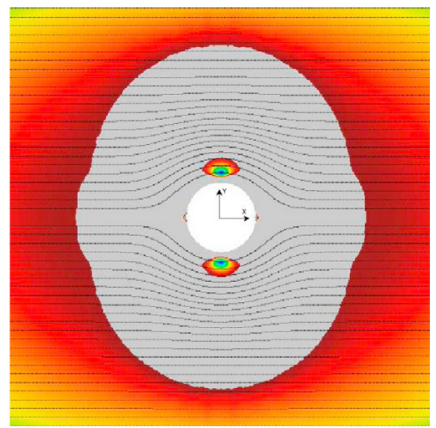

(b) $B n^{*}=100$
Fig. 16. Cylinder in an Herschel-Bulkley fluid. Yielded (grey) and unyielded (colored) regions and flow streamlines. Recirculation zones on $y$ axis and stagnation zones (with polar caps) on $x$ axis.

where $k$ is the consistency index of the fluid, $V$ is the velocity of the cylinder and $n$ is the flow index of the Herschel-Bulkley model.

\subsubsection{Model and results}

Fig. 12 shows the geometry and boundary conditions used in the current example. The geometry is similar to that considered in Section 6.2 , but in this case the semi-width of the domain, $L$, is taken sufficiently large not to influence the results. The minimum $L$ for this is smaller for Bingham than for Newtonian fluids and yet smaller for Herschel-Bulkley fluids.

The system of reference is fixed to the cylinder; therefore velocity boundary conditions are imposed on the external boundary of the domain (sides CE and ED in Fig. 12). A no slip boundary condition is imposed on the surface of the cylinder. The radius of the cylinder is $R=0.5 \mathrm{~m}$ and the velocity in the $x$ direction is $V=1 \mathrm{~m} / \mathrm{s}$ Due to the double symmetry of the problem, just a

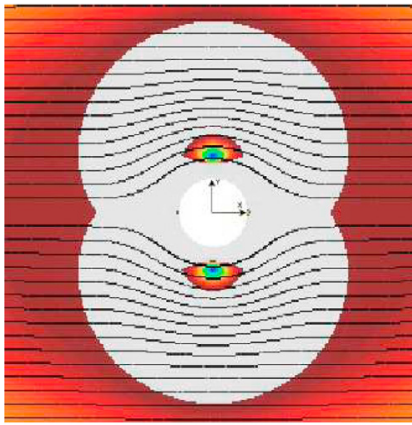

(a) $B n^{*}=10$

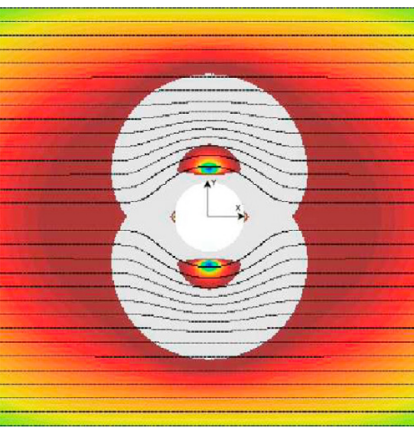

(b) $B n^{*}=100$
Fig. 18. Cylinder in a dilatant Herschel-Bulkley fluid $(n=2)$. Yielded (grey) and unyielded (colored) regions and flow streamlines. Recirculation region on $y$ axis and stagnation zone (with polar caps) on $x$ axis.

quarter of the domain is simulated and symmetry conditions are imposed.

The mesh used in the simulation is showed in Fig. 13.

Table 4 summarized the material properties of the model and the coefficients employed. Pseudoplastic $(n \leq 1)$ and dilatant Herschel-Bulkley fluids are considered. The particular case of Bingham plastics $(n=1)$ is also taken into account. A regularization coefficient $m=1000 \mathrm{~s}$ is used in all the simulations.

It can be observed in Fig. 14 that the non-dimensional drag coefficient $\left(F_{D}^{*}\right)$ grows with the flow index $n$, independently from the geometrical ratio, for $L: R \geq 50: 0.5$ (Table 5 ). This means that it is sufficient to consider a domain with that minimum geometrical ratio to ensure insensitivity of the flow from the artificial domain boundaries. It is evident form the results that the drag coefficient is linearly related to the flow index $n$ for $n \geq 0.5$.

The case of a pseudoplastic Herschel-Bulkley fluid is studied first. Fig. 15(a) and (b) present the non-dimensional drag, $F_{D}^{*}$, and

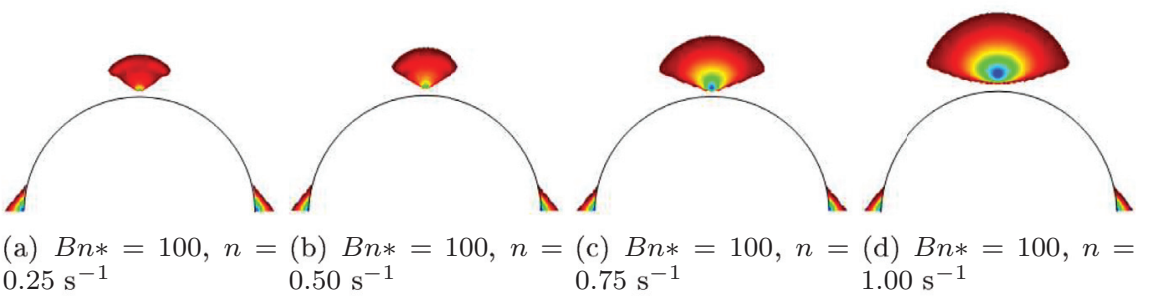

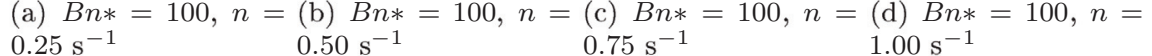

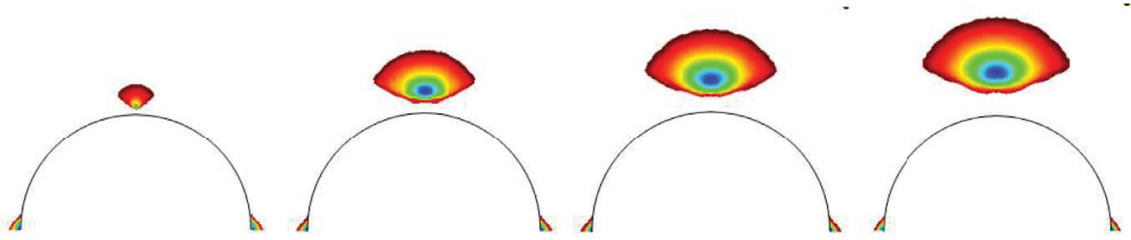

(e) $B n *=10, n=$ (f) $B n *=10, n=$ (g) $B n *=10, n=$ (h) $B n *=10, n=$

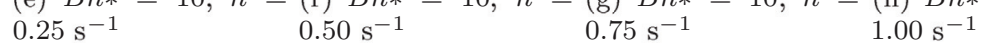

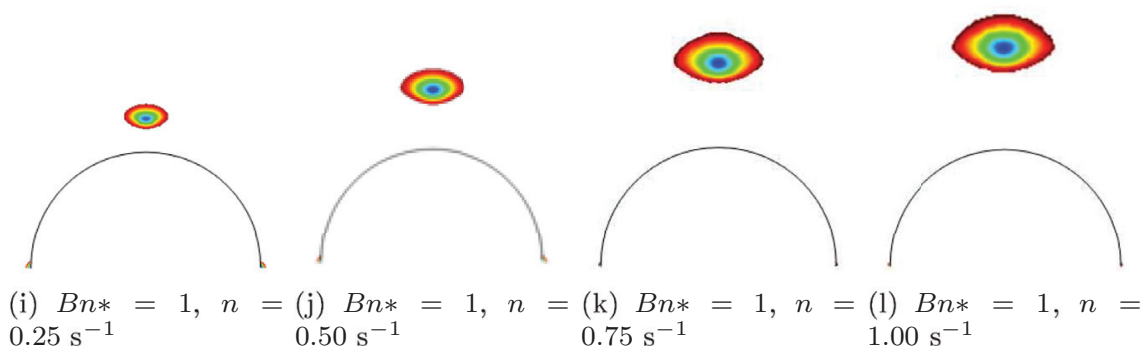

Fig. 17. Cylinder in an Herschel-Bulkley fluid. Dependency of the unyielded regions in terms of the Bingham number and the flow index. 

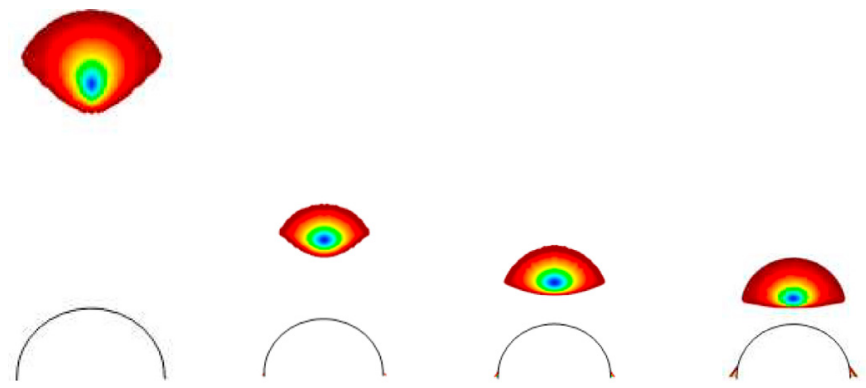

(a) $B n^{*}=0.1$

(b) $B n^{*}=1$

(c) $B n^{*}=10$

(d) $B n^{*}=100$

Fig. 19. Cylinder in a dilatant Herschel-Bulkley fluid $(n=2)$. Growth of the unyielded regions in terms of $B n^{*}$.

the drag force over the yield stress, $F_{D}^{\prime}=F_{D} / \tau_{y}$, respectively, versus the generalized Bingham number $\left(B n^{*}=0.1,1,10,100\right)$, for different flow indexes $(n=0.25,0.5,0.75,1)$. The drag coefficient grows as $B n^{*}$ increases (Fig. 15(a)) and the yield stress effect is higher for higher values of $B n^{*}$ (Fig. 15(b)).

The differences in the yielded and unyielded regions for different generalized Bingham numbers $\mathrm{Bn}^{*}$ are evident in Fig. 16 where the yielded region is plotted in grey for a $B n^{*}=10$ (Fig. 16(a)) and for a $B n^{*}=100$ (Fig. 16(b)). The increment of the $B n^{*}$ induces a shape and volumetric change of the yielded region which reduces significantly especially in the direction of the flow.

The stagnation and recirculation regions in terms of $B n^{*}$ and $n$ are shown in Fig. 17. The stagnation regions are very sensitive to the $B n^{*}$ while being almost insensitive to the value of the flow index $n$. In the stagnation region triangular shaped polar caps, similar to those obtained studying the falling of a sphere in [8], can be observed.

The recirculation zone on the $y$ axis increases when $\mathrm{Bn}^{*}$ or $n$ increase. The yielded thin layer between these regions and the cylinder reduces for higher values of $B n^{*}$, and increases with $n$. The no slip condition on the cylinder does not allow this "boundary layer" to disappear even for very high values of $\mathrm{Bn}^{*}$. The effect of an alternative slip boundary condition on the cylinder can be found in [36]. While the recirculation regions obtained match very well with those obtained by De Bresse in [36], the polar caps are significantly smaller. This is the consequence of the OSS stabilization technique used, that allows to solve with a high level of detail these critical parts of the domain.

The case of a dilatant Herschel-Bulkley fluid is considered next. The flow index is taken $n=2$. The magnitude of the velocity field

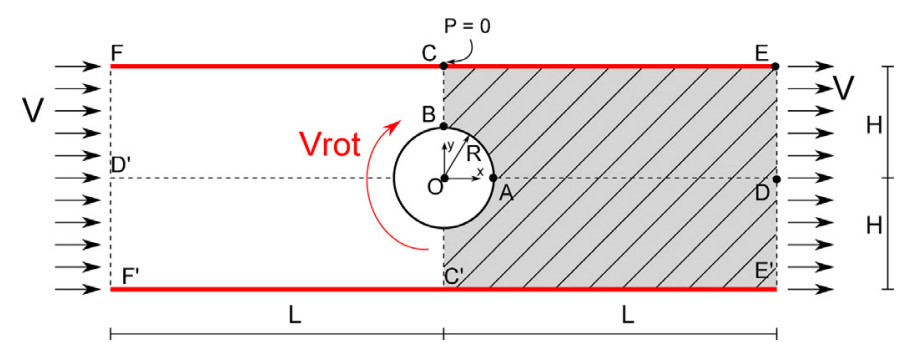

Fig. 21. Moving and rotating cylinder in an Herschel-Bulkley fluid. Geometry and boundary conditions.

Table 6

Moving and rotating cylinder in an Herschel-Bulkley fluid. Material parameters and regularization coefficient.

\begin{tabular}{lll}
\hline Material properties & & \\
\hline Consistency index & $k$ & $1 \mathrm{~Pa} \cdot \mathrm{s}^{n}$ \\
Yield stress & $\tau_{y}$ & $100 \mathrm{~Pa}$ \\
Flow index & $n$ & 0.25 \\
$\begin{array}{l}\text { Regularization } \\
\text { Regularization coefficient }\end{array}$ & $m$ & $1000 \mathrm{~s}$ \\
\hline
\end{tabular}

is smaller in the dilatant case than in the pseudoplastic one. As shown in Fig. 18, the yielded region has the shape of two circles intersected along the $x$ axis and it reduces when $B n^{*}$ increases much more faster than in the pseudoplastic case.

The polar caps start to be visible for $B n^{*} \geq 1$ while the recirculation regions are always present. These are bigger and more separated from the cylinder than in the corresponding pseudoplastic case (Fig. 19).

The drag coefficient in the dilatant case follows a similar dependency with $B n^{*}$ and $\tau_{y}$ as in the pseudoplastic case, but its absolute value is much lower (Fig. 20).

The shape of the stagnation and the recirculation regions are in good accordance with those obtained in [8] and [2] also, although in the latter the shape of the zones was more rounded.

\subsection{Flow around a moving cylinder rotating around its axis}

\subsubsection{Description of the problem}

The last example simulates a rotating cylinder moving between parallel planes in a Herschel-Bulkley fluid.

The principal objective is to study the yielded and unyielded region, to define the localization pattern of the strain rate and to see the evolution of the stream lines at different velocities of rotation.

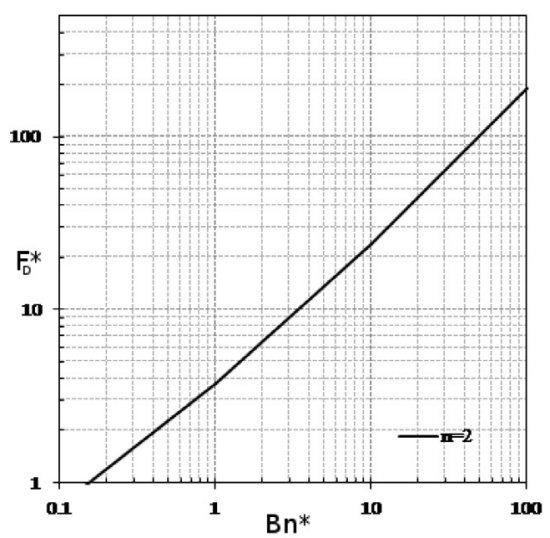

(a) Drag coefficient

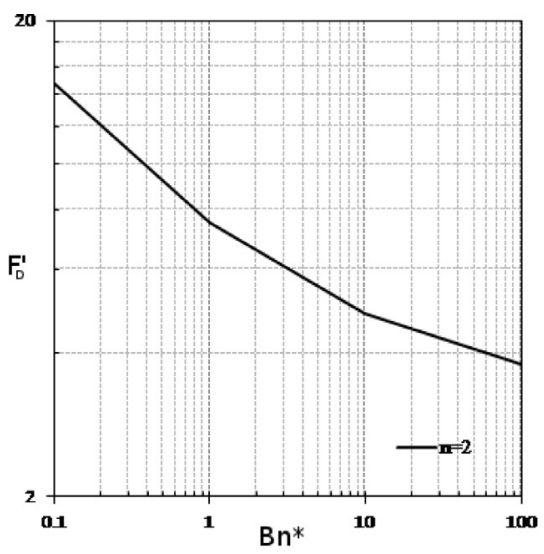

(b) Drag coefficient related to the yield stress

Fig. 20. Cylinder in a dilatant Herschel-Bulkley fluid $n=2$. Drag coefficient versus the Bingham number $B n^{*}$ and the yield stress. 


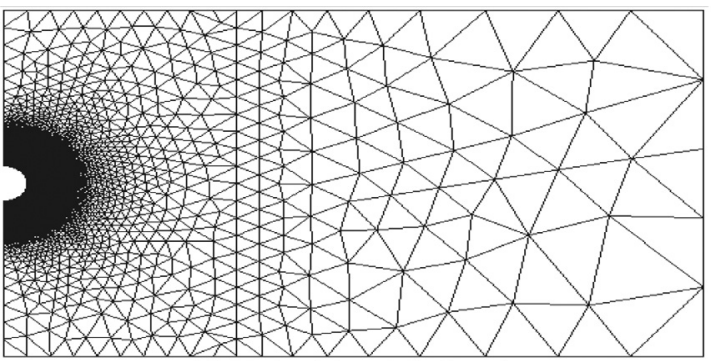

(a) Mesh of the whole domain

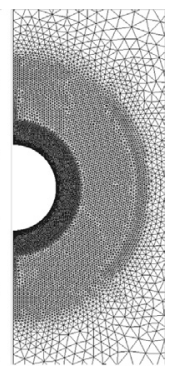

(b) Mesh around the cylinder
Fig. 22. Moving and rotating cylinder in an Herschel-Bulkley fluid. Unstructured mesh used for the calculation.

\subsubsection{Model and results}

The geometrical setting is similar to the one described in Section 6.2, but with the cylinder rotating around its axis. The problem is therefore antisymmetric with respect to the vertical axis $y$ (Fig. 21). This implies that only half of the domain needs to be simulated (the shaded area in Fig. 21), provided suitable boundary conditions are imposed on the plane of antisymmetry. The reference system is moving with the cylinder; therefore, on the outer boundary of the domain $u_{x}=V=1 \mathrm{~m} / \mathrm{s}$ is imposed in the $x$ direction, while $u_{y}=0 \mathrm{~m} / \mathrm{s}$. A no slip boundary condition is imposed on the surface of the cylinder.

Table 6 summarizes the properties of the material and the regularization parameter used. The flow index of the Herschel-Bulkley model is $n=0.25$, which corresponds to a highly pseudoplastic fluid.

The aspect ratio of the computational domain is $H: R=10: 1$ and $L: R=30: 1$. The unstructured mesh used in the example is shown in Fig. 22(a); it is composed of 9425 nodes and 18,345 linear triangular elements. The average size of the elements on the surface of the cylinder (see Fig. 22(b)) is of $0.01 \mathrm{~m}$, whereas on the vertical line (from $B$ to $C$ and from $G$ to $C^{\prime}$ in Fig. 21) the element size varies from $0.01 \mathrm{~m}$ to $0.04 \mathrm{~m}$.

Four different velocities of rotation $\left(V_{R O T}\right)$ have been studied: 0 , $0.5,1.0$ and $5 \mathrm{~m} / \mathrm{s}$. The symmetry with respect to the $x$ axis observed for $V_{R O T}=0 \mathrm{~m} / \mathrm{s}$ (Fig. 23(a)) is lost when the cylinder starts rotating. Under these circumstances only symmetry with respect to

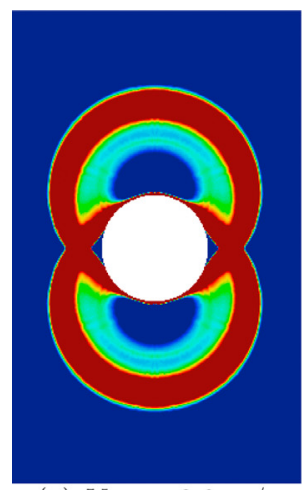

(a) $V_{\text {rot }}=0.0 \mathrm{~m} / \mathrm{s}$

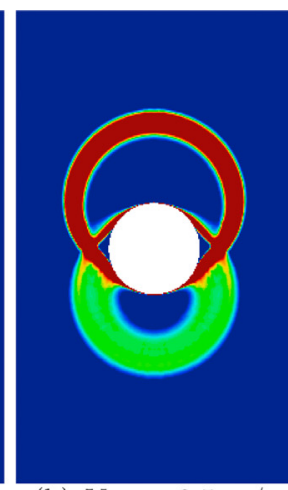

(b) $V_{\text {rot }}=0.5 \mathrm{~m} / \mathrm{s}$

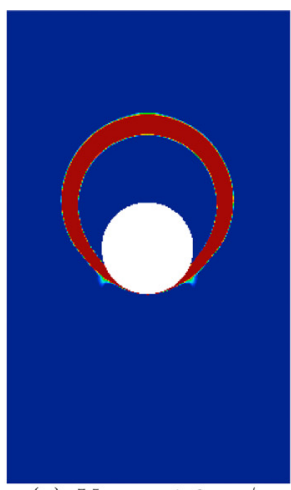

(c) $V_{\text {rot }}=1.0 \mathrm{~m} / \mathrm{s}$

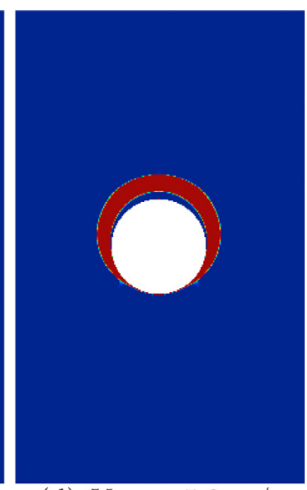

(d) $V_{\text {rot }}=5.0 \mathrm{~m} / \mathrm{s}$

Fig. 23. Moving and rotating cylinder in an Herschel-Bulkley fluid. Localization of strain rate for different rotational velocities.

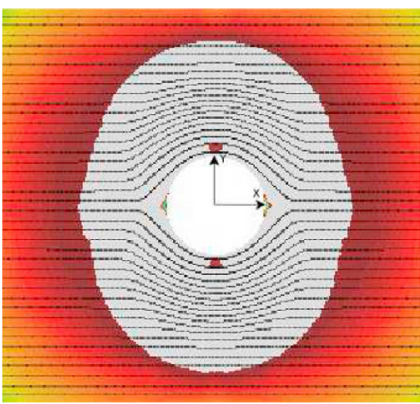

(a) $V_{\text {rot }}=0.0 \mathrm{~m} / \mathrm{s}$

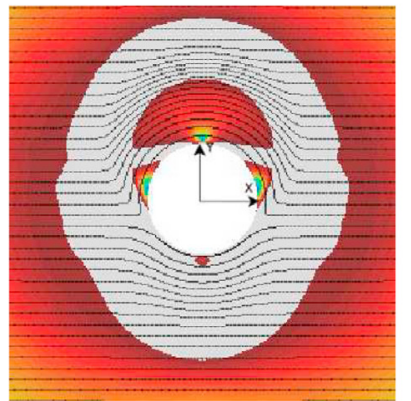

(c) $V_{\text {rot }}=1.0 \mathrm{~m} / \mathrm{s}$
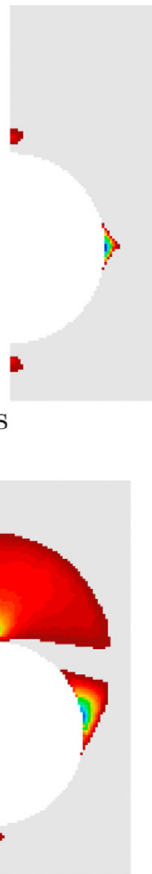

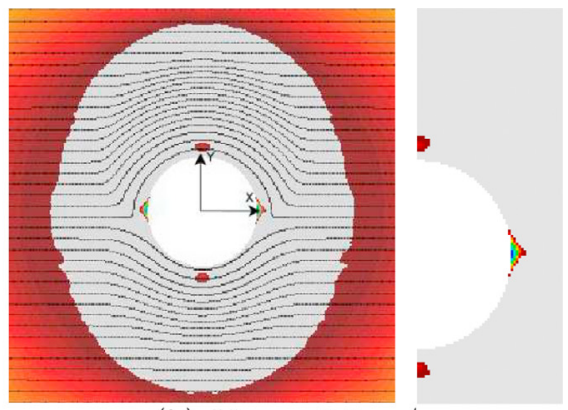

(b) $V_{\text {rot }}=0.5 \mathrm{~m} / \mathrm{s}$
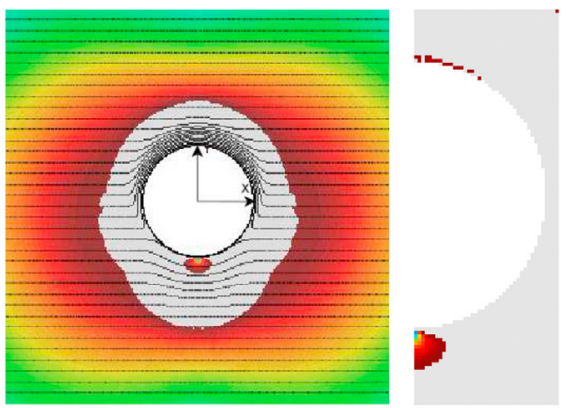

(d) $V_{\text {rot }}=5.0 \mathrm{~m} / \mathrm{s}$

Fig. 24. Moving and rotating cylinder in an Herschel-Bulkley fluid. Streamlines and yielded and unyielded regions for different rotational velocities. 


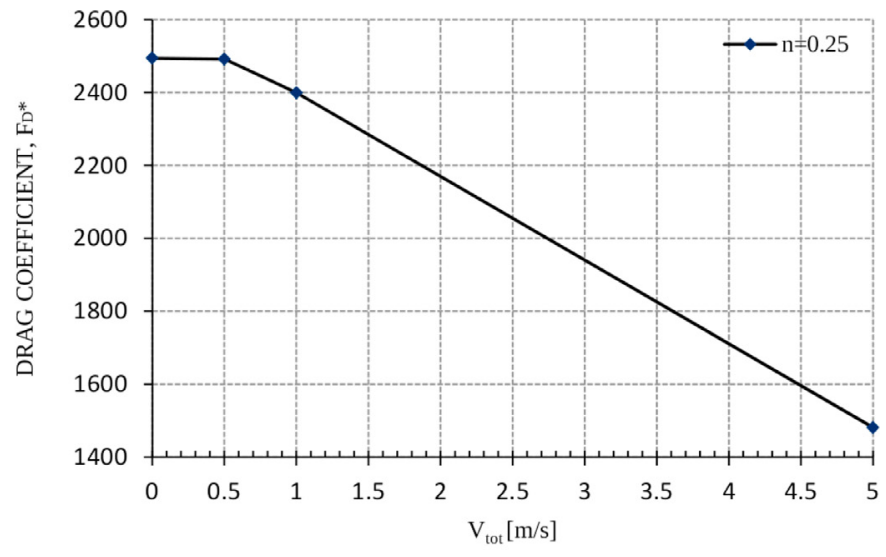

(a) Drag coefficient

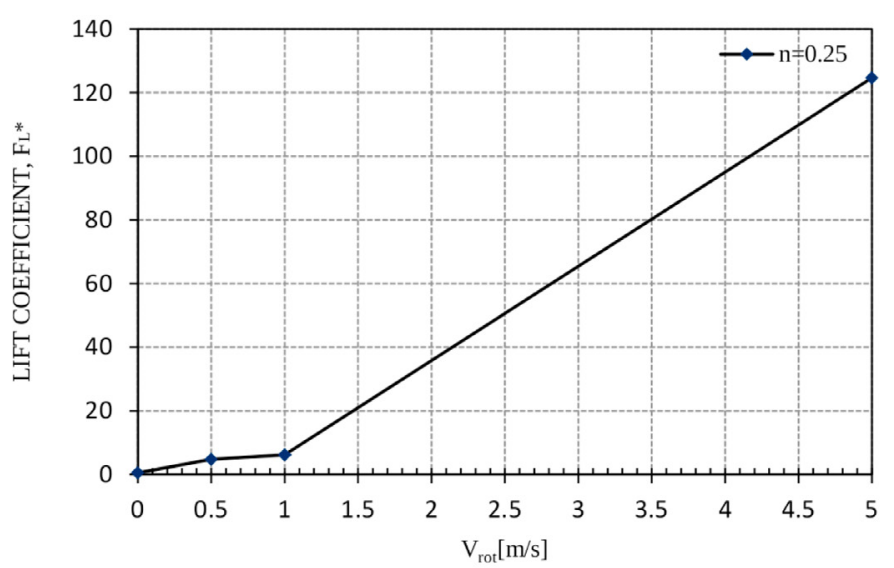

(b) Lift coefficient

Fig. 25. Moving and rotating cylinder in an Herschel-Bulkley fluid $n=0.25$. Drag and lift for different rotational velocities.

the vertical axis $y$ is maintained (Fig. 23(b)-(d)). This is confirmed by the streamlines (Fig. 24(a)-(d)).

The increment of the velocity of rotation makes one of the slip lines progressively disappear while the other moves closer to the cylinder. On one side of the cylinder the rotational velocity adds to the linear velocity, while it is opposed on the opposite side. For high values of the rotational velocity (Fig. 23(d)) the rate of strain localization concentrates around the cylinder.

The slip lines of Fig. 23 correspond to the change of slope in the streamlines (Fig. 24) that reduces their relative distance.

Fig. 24(a)-(d) shows the complex evolution of the yielded and unyielded regions as the velocity of rotation increases. The recirculation zone increases arriving to define a semi circle for $V_{R O T}=$ $1 \mathrm{~m} / \mathrm{s}$ and it disappears for $V_{R O T}=5 \mathrm{~m} / \mathrm{s}$, leaving a thin layer of unyielded material close to the surface while the size of the recirculation region under the cylinder increases. The growth of the stagnation region culminates for $V_{R O T}=1 \mathrm{~m} / \mathrm{s}$ and no polar caps are present for higher velocities.

The drag decreases as the velocity of rotation increases (Fig. 25). On the contrary, the lift coefficient, which is null when the cylinder is not rotating, increases with the velocity of rotation. It is worth noting that the drag is substantially higher than the lift in all the cases.

\section{Conclusions}

In the present work a mixed stabilized finite element formulation for Bingham and Herschel-Bulkley fluids is presented. The im- plementation of an OSS stabilization technique allows to use equal order interpolation of velocity and pressure (i.e., $P 1 / P 1$ linear elements), avoiding both the pressure and velocity oscillations and leading to a stable and accurate solution.

On the one hand, being OSS a residual based stabilization technique, no consistency error is introduced. On the other hand, constructing the subscale in the subspace orthogonal to the finite element one leads to a minimization of the numerical dissipation on the discrete solution.

The extrusion process of a Bingham fluid with the section reduced by $2 / 3$ shows a correct definition of the slip lines according to Pradtl's theory. A cylinder moving between two parallel planes is the second example studied. The comparison with the results obtained by other authors leads to the conclusion that the presented technique reproduces correctly the yielded and unyielded regions, as well as calculates the correct drag for different Bingham numbers and geometrical relations. Pseudoplastic and dilatant cases of Herschel-Bulkley are are also used to study a cylinder moving in an infinite domain and a cylinder moving and rotating around its axis. Also in these cases, the polar caps and recirculation regions are correctly reproduced.

\section{Acknowledgments}

The research was supported by the Spanish Ministry of Economy and Competitiveness (Ministerio de Economía y Competitividad, MINECO) through the project EACY (MAT2013-48624-C2-1-P). The financial support of the ERC Advanced Grant project SAFECON (AdG-267521) of the European Research Council and of the TMAPPP project (FP7 PEOPLE 2013 ITN-G.A.n607453) is also greatly acknowledged.

\section{References}

[1] S. Abdali, E. Mitsoulis, N. Markatos, Entry and exit flows of Bingham fluids, J. Rheol. 36 (2) (1992) 389-407.

[2] K. Adachi, N. Yoshioka, On creeping flow of a visco-plastic fluid past a circular cylinder, Chem. Eng. Sci. 28 (1) (1973) 215-226.

[3] J. Alexander, On complete solution for frictionless extrusion in plane strain, Quart. Appl. Math. 19 (1961) 31-40.

[4] D.D. Atapattu, R.P. Chhabra, P.H.T. Uhlherr, Creeping sphere motion in Herschel-Bulkley fluids: flow field and drag, J. Non-Newton. Fluid Mech. 59 (2) (1995) 245-265.

[5] D. Atapattu, R. Chhabra, P. Uhlherr, Wall effect for spheres falling at small Reynolds number in a viscoplastic medium, J. Non-Newton. Fluid Mech. 38 (1) (1990) 31-42, doi:10.1016/0377-0257(90)85031-S

[6] M. Beaulne, E. Mitsoulis, Creeping motion of a sphere in tubes filled with Herschel-Bulkley fluids, J. Non-Newton. Fluid Mech. 72 (1) (1997) 55-71.

[7] M. Bercovier, M. Engelman, A finite-element method for incompressible nonNewtonian flows, J. Comput. Phys. 36 (3) (1980) 313-326. http://dx.doi.org/10. 1016/0021-9991(80)90163-1.

[8] A. Beris, J. Tsamopoulos, R. Armstrong, R. Brown, Creeping motion of a sphere though a Bingham plastic, J. Fluid Mech. 158 (1985) 219-244.

[9] R.P. Bharti, R.P. Chhabra, V. Eswaran, Steady flow of power law fluids across a circular cylinder, Can. J. Chem. Eng. 84 (4) (2006) 406-421.

[10] E. Bingham, Fluidity and Plasticity, McGraw-Hill, New York, 1922.

[11] R. Bird, R. Armstrong, O. Hassager, Dynamics of Polymeric Liquids, Volume 1, Fluid Mechanics, 2nd Edition, John Wiley and Sons, New Yok, 1987.

[12] J. Blackery, E. Mitsoulis, Creeping motion of a sphere in tubes filled with a Bingham plastic material, J. Non-Newton. Fluid Mech. 70 (1-2) (1997) 59-77.

[13] F. Brezzi, M. Fortin, Mixed and Hybrid Finite Element Methods, Springer-Verlag, Berlin/New York, 1991.

[14] A. Brooks, T. Hughes, Streamline upwind/Petrov-Galerkin formulations for convection dominated flows with particular emphasis on the incompressible Navier-Stokes equations, Comput. Methods Appl. Mech. Eng. 32 (10̂3) (1982) 199-259. http://dx.doi.org/10.1016/0045-7825(82)90071-8.

[15] E. Castillo, R. Codina, Stabilized stress-velocity-pressure-finite element formulations of the Navier-Stokes problem for fluids with non-linear viscosity., Comput. Methods Appl. Mech. Eng. 279 (2014b) 554-578.

[16] E. Castillo, R. Codina, Variational multi-scale stabilized formulations for the stationary three-field incompressible viscoelastic flow problem, Comput. Methods Appl. Mech. Eng. 279 (2014a) 579-605, doi:10.1016/j.cma.2014.07.006.

[17] M. Cervera, M. Chiumenti, Size effect and localization in J2 plasticity, Int. J. Solids Struct. 46 (17) (2009) 3301-3312, doi:10.1016/j.ijsolstr.2009.04.025.

[18] M. Cervera, M. Chiumenti, R. Codina, Mixed stabilized finite element methods in nonlinear solid mechanics. part I: formulation, Comput. Methods Appl. Mech. Eng. 199 (2010) 2559-2570, doi:10.1016/j.cma.2010.04.006. 
[19] M. Cervera, M. Chiumenti, R. Codina, Mesh objective modeling of cracks using continuous linear strain and displacement interpolations, Int. J. Numer. Methods Eng. 87 (10) (2011) 962-987, doi:10.1002/nme.3148.

[20] M. Cervera, M. Chiumenti, C.A. de Saracibar, Shear band localization via local J2 continuum damage mechanics, Comput. Methods Appl. Mech. Eng. 193 (2004a) 849-880. http://dx.doi.org/10.1016/j.cma.2003.11.009.

[21] M. Cervera, M. Chiumenti, C. de Saracibar, Softening, localization and stabilization: capture of discontinuous solutions in J2 plasticity, Int. J. Numer. Anal. Methods Geomech. 28 (5) (2004b) 373-393, doi:10.1002/nag.341.

[22] M. Cervera, M. Chiumenti, Q.V.C.A. de Saracibar, Mixed linear/linear simplicial elements for incompressible elasticity and plasticity, Comput. Methods Appl. Mech. Eng. 192 (2003) 5249-5263, doi:10.1016/j.cma.2003.07.007.

[23] R.P. Chhabra, Bubbles, Drops, and Particles in Non-Newtonian Fluids,, Second Edition, CRC press Balkema, 2006.Print ISBN: 978-0-8247-2329-3 eBook ISBN: 978-1-4200-1538-6

[24] R.P. Chhabra, K. Rami, P.H.T. Uhlherr, Drag on cylinders in shear thinning viscoelastic liquids, Chem. Eng. Sci. 56 (6) (2001) 2221-2227.

[25] R. Chhabra, Encyclopedia of Fluid Mechanics,, Gulf, Houston, pp. 983-1033.

[26] R. Chhabra, J. Richardson, Non-Newtonian F and Applied Rheology, Engineering applications, 2008.

[27] M. Chiumenti, M. Cervera, R. Codina, A mixed three-field FE formulation for stress accurate analysis including the incompressible limit, Comput. Methods Appl. Mech. Eng. 283 (2015) 1095-1116, doi:10.1016/j.cma.2014.08.004.

[28] M. Chiumenti, Q. Valverde, C. Agelet De Saracibar, M. Cervera, A stabilized formulation for incompressible elasticity using linear displacement and pressure interpolations, Comput. Methods Appl. Mech. Eng. 191 (46) (2002) 5253-5264, doi:10.1016/S0045-7825(02)00443-7.

[29] R. Codina, Comparison of some finite element methods for solving the diffusion-convection-reaction equation, Comput. Methods Appl. Mech. Eng. $156(1-4)(1998)$ 185-210.

[30] R. Codina, On stabilized finite element methods for linear system of convection-diffusion-reaction equations, Comput. Methods Appl. Mech. Eng. 188 (2000a) 61-82.

[31] R. Codina, Stabilization of incompressibility and convection through orthogona sub-scales in finite element method, Comput. Methods Appl. Mech. Eng. 190 (2000b) 1579-1599.

[32] R. Codina, Stabilized finite element approximation of transient incompressible flows using orthogonal subscales., Comput. Methods Appl. Mech. Eng. 191 (2002) 4295-4321.

[33] R. Codina, O. Soto, Approximation of the incompressible Navier-Stokes equations using orthogonal subscale stabilization and pressure segregation on anisotropic finite element meshes, Comput. Methods Appl. Mech. Eng. 193 (2004) 1403-1419.

[34] A. Coppola, R. Codina, Improving Eulerian two-phase flow finite element approximation with discontinuous gradient pressure shape functions, Int. J. Numer. Methods Fluids 49 (2005) 1287.1304

[35] P. Coussot, Yield stress fluid flows: a review of experimental data, J. NonNewton. Fluid Mech. 211 (2014) 31-49.

[36] B.D. De Besses, A. Magnin, P. Jay, Viscoplastic flow around a cylinder in an infinite medium, J. Non-Newton. Fluid Mech. 115 (1) (2003) 27-49.

[37] E. Dean, R. Glowinski, G. Guidoboni, On the numerical simulation of Bingham visco-plastic flow: old and new results, J. Non-Newton. Fluid Mech. 142 (2007) 36-62.

[38] M. Diez, L. Godoy, Flujo viscoplástico incompresible de materiales con fricción y cohesión. aplicación a problemas bidimensionales, Revista Internacional de Métodos Numéricos para Cálculo en Ingeniería 7 (4) (1991) 417-436.

[39] D. dos Santos, S. Frey, M. Naccache, P. de Souza Mendes, Numerical approximations for flow of viscoplastic fluids in a lid-driven cavity, J. NonNewton. Fluid Mech. 166 (12-13) (2011) 667-679, doi:10.1016/j.jnnfm.2011.03. 004.

[40] G. Duvaut, J. Lions, Inequalities in Mechanics and Physics, Springer, 1976.

[41] I. Frigaard, C. Nouar, On the usage of viscosity regularisation methods for visco-plastic fluid flow computation, J. Non-Newton. Fluid Mech. 127 (2005) $1-26$.

[42] R. Glowinski, Numerical Methods for Nonlinear Variational Problems, Springer, 1984

[43] R. Glowinski, P.L. Tallec, Augmented Lagrangian and Operator-splitting Methods in Nonlinear Mechanics, SIAM,, Philadelphia, 1989.

[44] P.T. Griffiths, Flow of a generalised Newtonian fluid due to a rotating disk, J. Non-Newton. Fluid Mech. 221 (2015) 9-17, doi:10.1016/j.jnnfm.2015.03. 008.

[45] F. Händle, Extrusion in Ceramic, Springer, Berlin, 2007

[46] W. Herschel, R. Bulkley, Measurement of consistency as applied to rubberbenzene solutions, Proc. Am. Soc. Test. Mater. 26 (1926) 621-633.

[47] R. Hill, A theoretical analysis of the stresses and strains in extrusionand piercing, J. Iron Steel Inst., 159 (1948) 177-185.

[48] T. Hughes, Multiscale phenomena: Green's function, the Dirichlet to Neumann formulation, subgrid scale models, bubbles and the origins of stabilized formulations, Comput. Methods Appl. Mech. Eng. 127 (1995) 387-401.

[49] T. Hughes, G. Feijóo, L. Mazzei, J.-B. Quincy, The variational multiscale method-A paradigm for computational mechanics, Comput. Methods. Appl. Mech. Eng. 166 (1-2) (1998) 3-24.

[50] T. Hughes, L. Franca, G. Hulbert, A new finite element formulation for computational fluid dynamics: VIII. the Galerkin/least-squares method for advectivediffusive equations, Comput. Methods Appl. Mech. Eng. 73 (2) (1989) 173-189, doi:10.1016/0045-7825(89)90111-4.
[51] R. Huilgol, Fluid Mechanics of Viscoplasticity, Springer, Berlin Heidelberg, 2015.

[52] R. Huilgol, G. Kefayati, Natural convection problem in a Bingham fluid using the operator-splitting method, J. Non-Newton. Fluid Mech. 220 (2015) 22-32.

[53] R. Huilgol, Z. You, Prolegomena to variational inequalities and numerical schemes for compressible viscoplastic fluids, J. Non-Newton. Fluid Mech. 158 (2009) 113-126.

[54] L. Jossic, A. Magnin, Drag and stability of objects in a yield stress fluid, AIChE J. 47 (12) (2001) 2666-2672.

[55] A. Larese, E. Oñate, R. Rossi, A coupled Eulerian-PFEM Model for the Simulation of Overtopping in Rockfill Dams., Monograph CIMNE M133, 2012.ISBN: 978-84-940243-6-8, Deposito legal: B-29348-2012.1.

[56] A. Larese, R. Rossi, E. Oñate, M. Toledo, Physical and numerical modelization of the behavior of rockfill dams during overtopping scenarios, in: Proceedings of the Dam Maintenance and Rehabilitation II., CRCpress/Balkema, 2010, pp. 479487. ISBN:978-0-415-61648-5.

[57] A. Larese, R. Rossi, E. Oñate, S. Idelsohn, A coupled PFEM- Eulerian approach for the solution of porous FSI problems, Comput. Mech. 50 (6) (2012) 805-819, doi:10.1007/s00466-012-0768-9.

[58] A. Larese, R. Rossi, E. Oñate, Finite element modeling of free surface flow in variable porosity media., Arch. Numeri. Methods Eng. 22 (4) (2015) 637-653, doi:10.1007/s11831-014-9140-X.

[59] E. Lee, Numerical analysis of forming processes, Ed. J. Pitman O. Zienkiewicz R. Wood and J. Alexander, Jhon Wiley and Sons, Chichester, UK, 1984, pp. 373386.

[60] B.T. Liu, S.J. Muller, M.M. Denn, Convergence of a regularization method for creeping flow of a Bingham material about a rigid sphere, J. Non-Newton. Fluid Mech. 102 (2) (2002) 179-191.

[61] J. Lubliner, Plasticity Theory, Macmillan Publishing Company, New York, NY, 1990.

[62] J. Mandel, Ondes platiques dans un mileu indéfini á trois dimensions, J. Méc. 1 (1962) 3-30.

[63] O. Merkak, L. Jossic, A. Magnin, Spheres and interactions between spheres moving at very low velocities in a yield stress fluid, J. Non-Newton. Fluid Mech. 133 (2) (2006) 99-108.

[64] L. Minatti, A. Pasculli, SPH numerical approach in modelling 2D muddy debris flow, in: International Conference on Debris-Flow Hazards Mitigation: Mechanics, Prediction, and Assessment, 2011, pp. 467-475.

[65] K.A. Missirlis, D. Assimacopoulos, E. Mitsoulis, R.P. Chhabra, Wall effects for motion of spheres in power-law fluids, J. Non-Newton. Fluid Mech. 96 (3) (2001) 459-471.

[66] E. Mitsoulis, On creeping drag flow of a viscoplastic fluid past a circular cylinder: wall effects., Chem. Eng. Sci. 59 (4) (2004) 789-800.

[67] E. Mitsoulis, Annular extrudate swell of pseudoplastic and viscoplastic fluids, J. Non-Newton. Fluid Mech. 141 (2) (2007) 138-147.

[68] E. Mitsoulis, S. Galazoulas, Simulation of viscoplastic flow past cylinders in tubes, J. Non-Newton. Fluid Mech. 158 (1) (2009) 132-141.

[69] E. Mitsoulis, R. Huilgol, Entry flows of Bingham plastics in expansions, J. NonNewton. Fluid Mech. 122 (1-3) (2004) 45-54, doi:10.1016/j.jnnfm.2003.10.007.

[70] E. Mitsoulis, T. Zisis, Flow of Bingham plastics in a lid-driven square cavity, J. Non-Newton. Fluid Mech. 101 (2001) 173-180.

[71] M. Olshanskii, Analysis of semi-staggered finite-difference method with application to Bingham flows, Comput. Methods .Appl. Mech. Eng., 198 (2009) 975985.

[72] E. Oñate, La formulación del flujo viscoplástico y sus diversas aplicaciones prácticas por el método de los elementos finitos, Revista de Obras Públicas 127 (3178) (1980) 115-129.

[73] T.C. Papanastasiou, Flows of materials with yield., J. Rheol. 31 (1987) 385-404.

[74] D. Perić, S. Slijepcević, Computational modelling of viscoplastic fluids based on a stabilised finite element method., Eng. Comput. 18 (2001) 577-591.

[75] J.M. Piau, Viscoplastic boundary layer, J. Non-Newton. Fluid Mech. 102 (2) (2002) 193-218.

[76] R. Planas, S. Badia, R. Codina, Aproximation of the inductionless MHD problem using a stabilized finite element method, J. Comput. Phys. 230 (2011).29772966

[77] L. Prandtl, Uber dire harte plastischer korper, Gottinger Nachrichten (1920) 7485.

[78] A.M.V. Putz, T.I. Burghelea, I.A. Frigaard, D.M. Martinez, Settling of an isolated spherical particle in a yield stress shear thinning fluid, Phys. Fluids 20 (3) (2008). http://dx.doi.org/10.1063/1.2883937.

[79] A. Remaitre, J.P. Malet, O. Maquaire, C. Ancey, J. Locat, Flow behaviour and runout modelling of a complex debris flow in a clay-shale basin, Earth Surf. Process. Landf. 30 (4) (2005) 479-488.

[80] N. Roquet, P. Saramito, An adaptive finite element method for Bingham fluid flows around a cylinder, Comput. Methods Appl. Mech. Eng. 192 (31) (2003) 3317-3341.

[81] R. Rossi, A. Larese, P. Dadvand, E. Oñate, An efficient edge-based level set finite element method for free surface flow problems, Int. J. Numer. Methods Fluids 71 (6) (2013) 687-716, doi:10.1002/fld.3680.

[82] F. Salazar, J. Irazabal, A. Larese, E. Oñate, Numerical modelling of landslidegenerated waves with the particle finite element method (PFEM) and a nonNewtonian flow model, Int. J. Numer. Anal. Methods Geomech. (2015), doi:10. 1002/nag.2428.

[83] J. Sanchez F., Application of first-order operator splitting method to Bingham fluid flow simulation, Comput. Math. Appl. 36 (3) (1998) 71-86. 
[84] P. Sivakumar, R. Bharti, R. Chhabra, Effect of power-law index on critical parameters for power-law flow across an unconfined circular cylinder, Chem. Eng. Sci. 61 (18) (2006) 6035-6046, doi:10.1016/j.ces.2006.05.031.

[85] S. Slijepcević, D. Perić, Some aspects of computational modelling of nonNewtonianfluids based on a stabilised finite element method, in: Proceeding of the ECCOMAS 2004 - European Congress on Computational Methods in Applied Sciences and Engineering, 2004

[86] H. Tabuteau, P. Coussot, J. De Bruyn, Drag force on a sphere in steady motion through a yield-stress fluid, J. Rheol. 51 (1) (2007) 125-137, doi:10.1122/1. 2401614.

[87] R.I. Tanner, Stokes paradox for power-law flow around a cylinder, J. NonNewton. Fluid Mech. 50 (2) (1993) 217-224.

[88] R. Tanner, J. Milthorpe, Numerical simulation of the flow of fluids with yield stresses, Proceedings of the Third International Conference on Numerical Methods in Laminar and Turbulent Flow: 1983, Eds: C. Taylor J.A. Johnson W.R. Smith, Swansea, UK 1983, pp. 680-690.
[89] M. Van Dyke, Perturbation Methods in Fluid Mechanics (Vol. 964), New York: Academic Press, 1964.

[90] K. Walters, R.I. Tanner, The motion of a sphere through an elastic fluid., Transport Processes in Bubbles, Drops, and Particles Eds. R.P. Chhabra and D. DeKee, Hemisphere, New York, NY (1992).

[91] N. Yoshioka, K. Adachi, On variational principles for a non-Newtonian fluid, J. Chem. Eng. Jpn. 4 (3) (1971) 217-220.

[92] J. Zhang, An augmented Lagrangian approach to Bingham fluid flows in a liddriven square cavity with piecewise linear equal-order finite elements, Comput. Methods Appl. Mech. Eng. 199 (2010) 3051-3057, doi:10.1016/j.cma.2010. 06.020.

[93] O. Zienkiewicz, P. Jain, E. Oñate, Flow of solids diuring forming and extrusion: some aspects of numerical solutions, Int. J. Solids Struct. 14 (1978) 15-38.

[94] T. Zisis, E. Mitsoulis, Viscoplastic flow around a cylinder kept between parallel plate, J. Non-Newton. Fluid Mech. 105 (1) (2002) 1-20. 